\section{OPEN JOURNAL SYSTEMS}

ISSN:2237-2202
Available on line at Directory of Open Access Journals

Journal of Hyperspectral Remote Sensing v.9, n.2 (2019) 49-67

www.periodicos.ufpe.br/revistas/jhrs

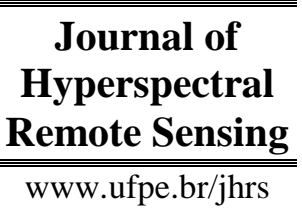

\title{
Different statistical methods for the discrimination of tropical mangrove species using in-situ hyperspectral data
}

\author{
Tanumi Kumar ${ }^{*}$, Dibyendu Dutta ${ }^{* *}$, Diya Chatterjee ${ }^{* * *}$, K Chandrasekar $^{* *}$, Goru S. Rao ${ }^{* * * *}$, Uday Raj ${ }^{* * * *}$ \\ "Regional Remote Sensing Centre-East, National Remote Sensing Centre, Indian Space Research Organisation, \\ Department of Space, Government of India, BG-2, Action Area- 1B, New Town, Kolkata 700156, India, E-mail: \\ tanumi_k@nrsc.gov.in; tanumikumar@yahoo.co.in (Corresponding author) \\ ***National Remote Sensing Centre, Indian Space Research Organisation, Department of Space, Government \\ of India, Annaram Village Road, Shadnagar 509216, Telangana, India \\ ***:Department of Geo-Informatics, Sangam University, Rajasthan 311 802, India \\ ****: Indian Space Research Organisation HQ (ISRO HQ), Department of Space, Government of India, Antariksh \\ Bhavan, New BEL Road, Bengaluru 560231, India \\ National Remote Sensing Centre, Indian Space Research Organisation, Department of Space, Government of \\ India, \\ Balanagar, Hyderabad 500037, India
}

Received 19 April 2019, accepted 9 May 2019

\begin{abstract}
The study highlights the hyperspectral characteristics of canopies of 14 tropical mangrove species, belonging to nine families found in the tidal forests of the Indian Sundarbans. Hyperspectral observations were recorded using a field spectroradiometer, pre-processed and subjected to derivative analysis and continuum removal. Mann-Whitney U tests were applied on the spectral data in four spectral forms: (i) Reflectance Spectra (ii) First Derivative, (iii) Second Derivative and (iv) Continuum Removal Reflectance Spectra. Factor analysis was applied in each of the spectral forms for feature reduction and identification of the important wavelengths for species discrimination. Stepwise discriminant analysis was used on the feature reduced reflectance spectra to obtain optimal bands for computation of Jeffries-Matusita distance. The Mann-Whitney U test could be satisfactorily used for determining the significant (separable) bands for discriminating the species. In general, the red region, red edge domain, specific near infrared bands (including 759, 919, 934, 940, 948, 1152, 1156, 1159 and $1212 \mathrm{~nm}$ ) and shortwave infrared region (1503-1766 nm) played major roles in spectral separability. Overall, hyperspectral data showed potential for discriminating between mangrove canopies of different species and the results of the study also indicated the usefulness of the applied statistical tools for discrimination.

Keywords: mangrove species, in-situ hyperspectral data, statistical methods, species discrimination
\end{abstract}

\section{Introduction}

Mangrove vegetation comprises a variety of salt tolerant species growing in the intertidal areas between the land and the sea (Reddy et al., 2007). Mangroves are taxonomically a very diverse assemblage of woody spermatophytes, typically occurring along tropical coastlines in the saline environment under tidal influence (Snedaker, 1982). Tomlinson (1986) expressed the term 'mangrove' as the intertidal ecosystem that is highly adapted to thrive in the coastal environment. Mangrove genera and families exhibit certain morphological, biological, physiological and ecological adaptations. The most prominent adaptations are pneumatophores (e.g. Avicennia sp., Sonneratia sp.), stilt roots (e.g. Ceriops sp., Rhizophora sp., Bruguiera sp.), salt glands and viviparous germination (Giri et al., 2014). The mangrove species composition and distribution depends on some environmental factors like soil composition, duration and frequency of the tidal inundation (Kuenzer et al., 2011).

The continuous loss of mangroves all over the world and consequent quest for conservation initiative activities lead to the mapping and monitoring of mangrove forests (Blasco and Aizpuru 2002; Huitric et al., 2002). Remote sensing has emerged as a powerful tool for investigation and mapping of mangrove areas using earth observation satellite data collected in the last few decades; mangroves have been mapped both in the global (Giri et al., 2011) as well as in the regional (India) scales (e.g. Reddy et al., 2007; State of Forest Report, 2011; Ajai et al., 2012). 
Broadband multispectral remote sensing has been found to be insufficient to differentiate the mangrove classes at genus or species rank (Holmgren and Thuresson, 1998). The potential of hyperspectral imaging and image processing has already been demonstrated for various applications in vegetation structure, composition and physiology (Van Der Meer, 2001; Apan and Phinn, 2006). This benefit is mostly determined by its capability to measure reflectance and absorption in explicit and narrow spectral bands. Airborne and space-borne satellite hyperspectral data offer a large number of narrow, contiguous, spectral bands, over the 400 to $2500 \mathrm{~nm}$ range of the electromagnetic spectrum (Van Der Meer, 2001; Apan and Phinn, 2006). Each pixel in the image has an associated spectrum comparable to the spectra of the material acquired in the laboratory or from the field. As a result, hyperspectral data allow for an improved segregation of feature types based on their distinctive spectral reflectance and absorption characteristics (Hirano, 2003; Demuro, 2003; Jensen, 2005). Several studies have been carried out in the past years on mangrove forests using hyperspectral data to extract more information from continuous signatures acquired through narrow wavelength intervals (Held et al., 2003; Hirano et al., 2003; Koedsin et al., 2013). The efficacy of hyperspectral data (airborne) for species level classification of mangrove forests of Port Klang, Selangor, Malaysia has shown promising outcomes (Jusoff, 2006). The potential of CASI-2 data was investigated for Australian mangrove species mapping using pixel based and object-based image approaches by Kamal and Phinn (2011). Moreover, an Earth Observing-1 (EO-1) Hyperion image of the mangrove area of Bhitarkanika National Park, Odisha, India was used by Kumar et al. (2013) for classification of the mangroves into floristic composition classes; amongst the three full-pixel classifiers tested in the investigation, Support Vector Machine produced the best results in terms of training pixel accuracy. The work of Chakravortty (2013) mainly focused on the application of hyperspectral data for development of a spectral library of mangrove species; the author established the potential of hyperspectral imagery for species level classification of mangroves in the Henry Island of Sundarban Biosphere Reserve, India.

Various works demonstrated the use of leaf hyperspectral reflectance measurements. Use of Jeffries-Matusita (J-M) distance indices for spectral separation of leaves of some mangroves species has been reported where a genetic search algorithm-based selector was used for choosing a subset of bands that retained spectral separability between classes of mangrove species at Sawi Bay, Chumporn Province, Thailand (Vaiphasa et al., 2005). Kamaruzaman and
Kasawani (2007) carried out discrimination of four mangrove species and one mangrove associate from Tok Bali, Malaysia, using hyperspectral foliar reflectance measurements. Comparable research was conducted by Wang and Sousa (2009) for discerning three mangrove species from the Caribbean coast of Panama; the authors also used ratio indices for detecting stress in these mangroves. Leaves of four mangrove species of the West Bengal Sundarbans and the Gulf of Kachchh (India) were discriminated by Panigrahy et al. (2012) using various statistical approaches. Zhang et al. (2014) recognized the state (healthy/ poor condition) of mangroves in a Mexican coastal lagoon using proximal hyperspectral remote sensing methods (including $\mathrm{R}^{2}$ plot, principal component analysis and stepwise discriminant analysis). The potential of in-situ hyperspectral data for discriminating mangroves of nine families and for discerning mudflat classes of the Indian Sundarbans was studied by Manjunath et al. (2013); in this study the authors used parametric tests for spectral discrimination. Laboratory leaf spectra of Bhitarkanika, Odisha mangroves were analyzed by Prasad and Gnanappazham (2014, 2015 and 2016) who carried out derivative spectral analysis and several statistical approaches for discriminating between eight species of Rhizophoraceae family. Considering the numerous existing statistical approaches, it is often difficult to choose the most appropriate data analysis method or group of methods for differentiating spectral signatures. The present study aims to test some of the commonly available statistical methods in the context of in-situ canopy hyperspectral data for mangrove species discrimination. Thus, the objectives of the work were: (1) evaluation of the utility of hyperspectral data for discrimination of canopies of mangrove species using field hyperspectral data and (2) exploration and comparison of different statistical approaches to harness essential information from the collected data.

\section{Materials and methods}

\section{Study area}

The Indian Sundarbans mangrove ecosystem $\left(21^{\circ} 31^{\prime} \mathrm{N}\right.$ to $22^{\circ} 15^{\prime} \mathrm{N}$ and $88^{\circ} 10^{\prime} \mathrm{E}$ to $\left.89^{\circ} 09^{\prime} \mathrm{E}\right)$ lies in the delta of two rivers, the Ganges and the Brahmaputra of the Indian subcontinent. Around 37 species of obligate mangroves and 32 mangrove associates have been reported in this area (Banerjee et al., 1989). The study area of this work was the mangrove habitat of the Jharkhali area $\left(21^{\circ} 57^{\prime} 06^{\prime \prime} \mathrm{N}\right.$ to $22^{\circ} 06^{\prime} 23^{\prime \prime} \mathrm{N}$ and $88^{\circ} 37^{\prime} 07^{\prime \prime} \mathrm{E}$ to $88^{\circ} 48^{\prime} 20^{\prime \prime} \mathrm{E}$ ) (Figure 1). Jharkhali is located at the estuary of the Matla-Bidya river system. The targets for spectral measurements were canopies of different mangrove 
species. The selection of Jharkhali was based on the fact that the area harbours diverse species of mangroves. Altogether, 14 mangrove tree species

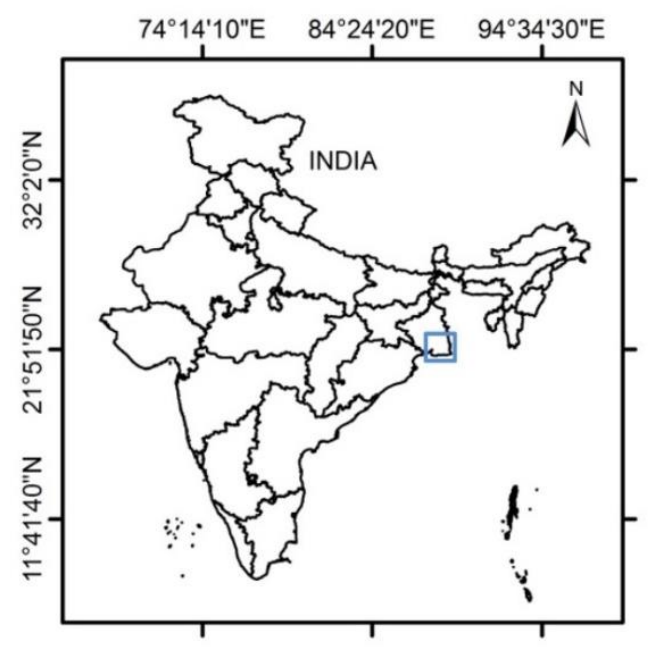

(from both natural forests and plantations) belonging to nine families were explored in this study (Table 1).
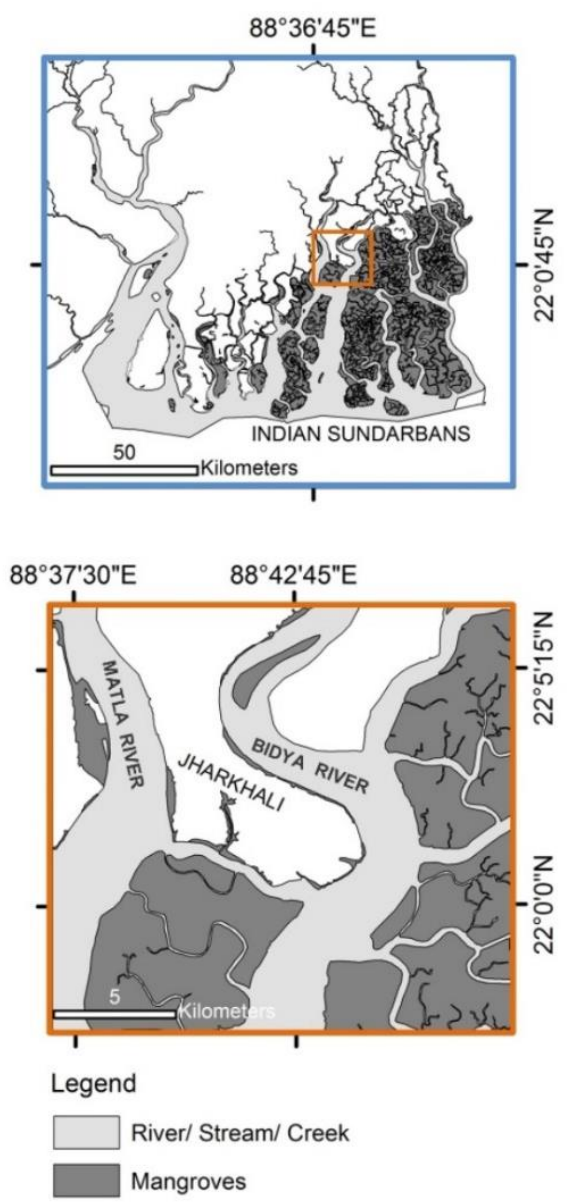

Figure 1 - Location map of Jharkhali, Indian Sundarbans.

Table 1. List of mangrove species covered under this study.

\begin{tabular}{lll}
\hline Scientific Name & Abbreviation & Family \\
\hline Avicennia alba Blume & $\mathrm{Aa}$ & Arecaceae \\
Aegiceras corniculatum (L.) Blanco & $\mathrm{Ac}$ & Mysinaceae \\
Avicennia marina (For.) Vierh & $\mathrm{Am}$ & Avicenniaceae \\
Avicennia officinalis L. & $\mathrm{Ao}$ & Avicenniaceae \\
Aegialitis rotundifolia Roxburgh & $\mathrm{Ar}$ & Plumbaginaceae \\
Bruguiera gymnorrhiza (L.) Lamk & $\mathrm{Bg}$ & Rhizophoraceae \\
Ceriops decandra (Griff.) Ding Hou & $\mathrm{Cd}$ & Rhizophoraceae \\
Excoecaria agallocha L. & $\mathrm{Ea}$ & Euphorbiaceae \\
Heritiera fomes (Perr.) Robbins & $\mathrm{Hf}$ & Sterculiaceae \\
Lumnitzera racemosa Willd. & $\mathrm{Lr}$ & Combretaceae \\
Nypa fruticans Wurmb & $\mathrm{Nf}$ & Arecaceae \\
Phoenix paludosa Roxb. & $\mathrm{Pp}$ & Arecaceae \\
Rhizophora mucronata Lamk. & $\mathrm{Rm}$ & Rhizophoraceae \\
Xylocarpus granatum Koen. & $\mathrm{Xg}$ & Meliaceae \\
\hline
\end{tabular}




\section{Spectral measurements}

Spectral reflectance measurements of canopies of 14 mangrove species were taken during low tide conditions during February-March 2016 and on cloud-free days between 10 to $11: 30 \mathrm{hrs}$ (local time). Trees were randomly selected based on the availability of the mangrove species. Measurements were taken using a hyperspectral spectroradiometer (HR 1024) having 971 channels in the range of 350$2500 \mathrm{~nm}$. The sensor, equipped with a field-of-view (FOV) $14^{\circ}$, was positioned $1 \mathrm{~m}$ above the target at the nadir position. The sampled trees were approximately $1.5 \mathrm{~m}$ above the ground (about $1 \mathrm{~m}$ for Phoenix paludosa and Nypa fruticans). The diameter of the observation field or the ground range was about $25 \mathrm{~cm}$; thus, the FOV would cover a small section of a branch. However, during measurements, it was ensured that the FOV was fully covered by leaves of the concerned species. Moreover, since the tree species were more than 10 years old and had good canopy cover with multiple layers, the background (soil/ water) was more or less completely covered with leaves. Hence, the contribution from background on the reflectance was negligible for most of the species. Nevertheless, owing to the presence of large compound leaves in P. paludosa and $N$. fruticans, the background effect was unavoidable in these two species. Measurements were collected from the sunlit sides of the tree canopies to maximize the incident light intensity. A reference measurement was collected with a white
Spectralon reference panel before and after each target measurement. Dark current measurements were automatically taken immediately prior to the reference or target scans. The equipment was set up to use an average of ten scans to represent a single observation and 10 observations were obtained for each tree per mangrove species.

\section{Hyperspectral data processing and data treatments \\ Measurements near the water absorption} bands and the extreme shortwave infrared (SWIR) bands yielded only noisy data. As a result, in the present study, spectral bands from $350 \mathrm{~nm}$ to 1346 $\mathrm{nm}, 1489 \mathrm{~nm}$ to $1766 \mathrm{~nm}$ and $2022 \mathrm{~nm}$ to $2281 \mathrm{~nm}$ were used for further analysis. However, bands between 350 and $390 \mathrm{~nm}$ were not included in the results because usually this part of the wavelength range in satellite data analysis is not considered as it is affected by haze. Thus, a total of 724 bands have been shown in the results and discussed. The instrument provides a sampling interval of $1.3 \mathrm{~nm}$ for the spectral region $350-980 \mathrm{~nm}, 3.6 \mathrm{~nm}$ for $983-$ $1766 \mathrm{~nm}$ and $2.5 \mathrm{~nm}$ for $2022-2281 \mathrm{~nm}$.

The above data were subjected to various forms of data transformation, statistical analysis and feature reduction using statistical software SPSS 16.0 and ENVI 4.7. Graphs were prepared and analyzed using Origin Pro 8. The methodology adopted to assess the separability of the species spectrally is schematically represented in Figure 2.

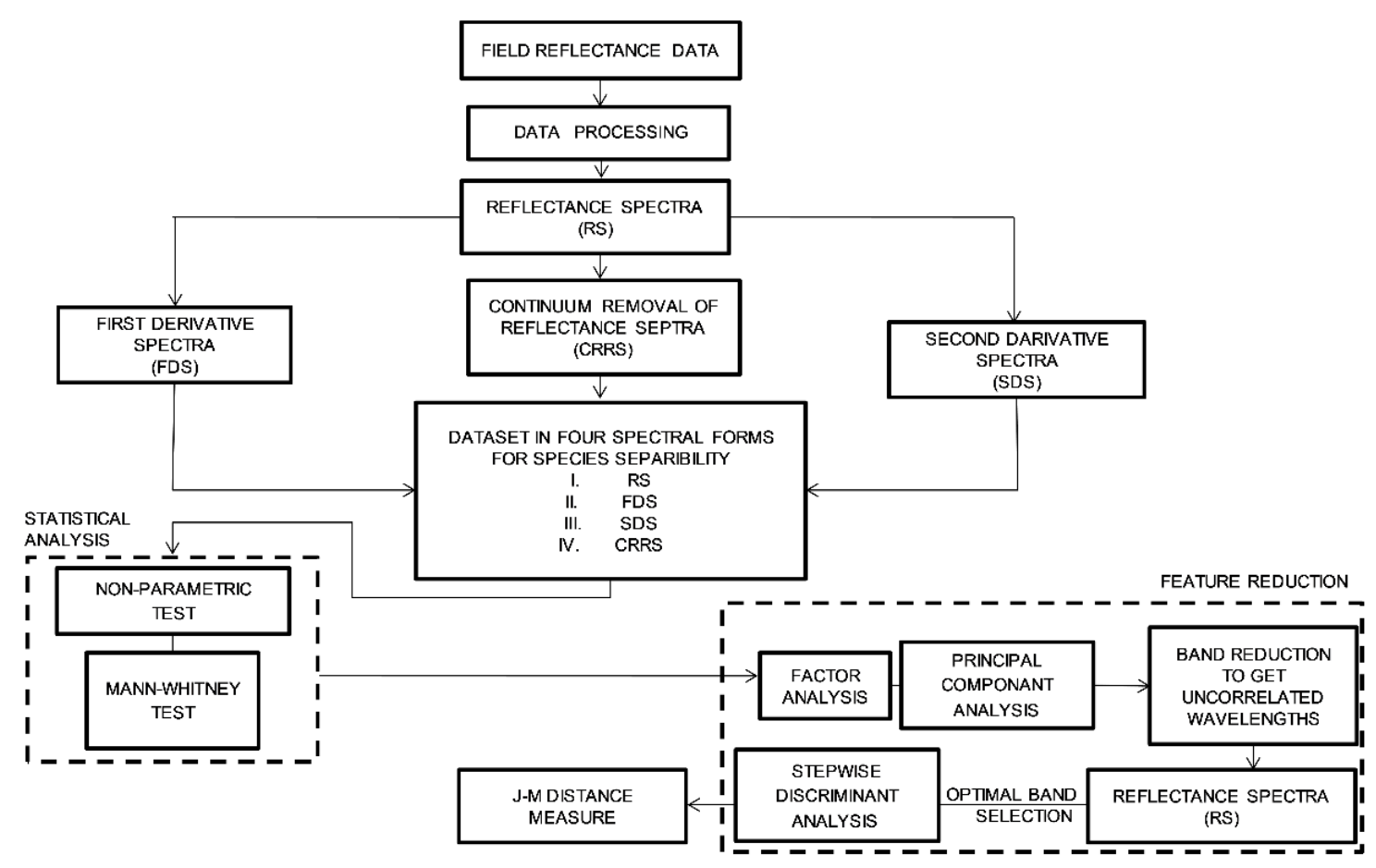

Figure 2 - Methodology flowchart. Abbreviations as in the text. 
Derivative spectral analysis

Spectral derivative analysis was done by dividing the difference between successive spectral values by its wavelength interval (bandwidth). Finite approximation method could be used to estimate derivatives based on the spectral resolution of the data (i.e., bandwidth) $\Delta \lambda$ (Tsai and Philpot, 1998). The formula used to estimate the first derivative is

$$
\left.\frac{d s}{d \lambda}\right|_{i} \approx \frac{s\left(\lambda_{i}\right)-s\left(\lambda_{j}\right)}{\Delta \lambda}
$$

Where, $\Delta \lambda$ is the band width which is given as $\Delta \lambda \approx \lambda$ ${ }_{\mathrm{i}}-\lambda_{\mathrm{j}}$ and also $\lambda_{i}>\lambda_{j}$. In the study first derivative spectra have been designated as FDS.

The second derivative was calculated from the first derivative and it could be expressed as

$$
\left.\left.\frac{d^{2} y}{d x^{2}}\right|_{j} \approx \frac{d}{d \lambda}\left(\frac{d s}{d \lambda}\right)\right|_{j} \approx \frac{s\left(\lambda_{i}\right)-2 s\left(\lambda_{j}\right)+\left(\lambda_{k}\right)}{(\Delta \lambda)^{2}}
$$

Where, $\Delta \lambda=\lambda_{k}-\lambda_{j}=\lambda_{j}-\lambda_{i}$ and $\lambda_{k}>\lambda_{j}>\lambda_{i}$. In the study second derivative spectra have been designated as SDS.

Continuum removal of reflectance spectra

Continuum removal method is generally used to enhance the absorption region of spectrum. In recent times, this method was applied in vegetationrelated studies such as species discrimination and to correlate pigment content with spectra (Schmidt and Skidmore, 2003; Mutanga and Skidmore, 2007; Sun et al., 2008). It is a normalization technique with the values ranging between 0 and 1 with absorption features more emphasized and variability in absolute reflectance being eliminated. In the present study area 'convex hull' method was used, which connected the local maxima of the spectrum as the rubber band was stretched and connected in maximum reflectance points. The continuum line was drawn by connecting the consecutive local maxima. Then it transformed the selected local maxima to the maximum reflectance value of 1 and thereby, the absorption features are enhanced (Clark et al., 1987). Continuum removal was performed in ENVI 4.7. Continuum removed reflectance spectra have been designated as CRRS.

\section{Statistical test}

In the present study non-parametric statistical analysis has been carried out to identify the bands having separability. In a parametric test, such as the $t$ test, F-test, Analysis of Variance (ANOVA) etc., there is a requirement of an assumption about the population. For example, in $t$-test it is assumed that the populations are normally distributed. On the other hand, non-parametric tests do not require any assumption regarding the population.

In the present study, non-parametric MannWhitney U tests were applied on reflectance spectra (RS), first derivative spectra (FDS), second derivative spectra (SDS) and continuum removed reflectance spectra (CRRS) of 91 possible pairs of species combinations.

\section{Mann-Whitney U test}

Mann-Whitney $U$ test compares median spectral reflectance of two species to find out the spectral difference between them (Dineen and Blakesley, 1973) at each wavelength location. This test does not assume normal distribution with null hypothesis $\mathrm{H}_{0}: \mathrm{n}_{1}=\mathrm{n}_{2}$, against the alternate hypothesis, $\mathrm{H}_{\mathrm{a}}: \mathrm{n}_{1} \neq \mathrm{n}_{2}$. The data were ranked (in ascending order) and it follows $Z$ table as the sample size is above 20. The test statistic used for the calculation was

$$
Z=\frac{\left(U-\mathrm{n}_{1} \mathrm{n}_{2} / 2\right)}{\sqrt{\frac{\mathrm{n}_{1} \mathrm{n}_{2}}{N(N-1)}}-\left(\frac{N^{3}-N}{12}-\sum_{i} T_{i}\right)}
$$

Where,

$U \quad U$ statistic, $U=\mathrm{n}_{1} \mathrm{n}_{2}+\mathrm{n}_{1}\left(\mathrm{n}_{1}+1\right) / 2-S_{1}$

$S \quad$ sum of ranks of particular group

$\mathrm{n}_{1} \& \mathrm{n}_{2}$ the number of observations in each group

$N \quad$ the total number of observations

$T_{i} \quad$ sum of ranks for tied observations, $T_{i}=$ $t_{3}-t / 12$

$t \quad$ number of observations tied for rank $I$.

Though all the statistical tests were conducted in both 95 and $99 \%$ confidence intervals, results have been given only for $99 \%$ confidence interval as they are more sensitive.

\section{Feature reduction analysis}

This was performed to obtain uncorrelated wavelengths out of the total number of wavelengths selected after the statistical tests in each of the four spectral forms.

Factor analysis

The RS, FDS, SDS and CRRS data were separately subjected to factor analysis, which attempts to identify underlying variables (here wavelengths) that explain the pattern of correlations within a set of observed variables. In this work, this analysis was used for data reduction and as a tool to identify the wavelengths that were uncorrelated and contained maximum information. The method used for 
extraction was principal component analysis (PCA), which forms uncorrelated linear combinations of the observed variables. The first component has maximum variance. Successive components explain progressively smaller portions of the variance and are all uncorrelated with each other. The technique adopted for rotation was Varimax method with Kaiser Normalization, which is an orthogonal rotation method that minimizes the number of variables that have high loadings on each factor (Norusis, 2004). This method simplifies the interpretation of the factors. Eigenvalues over one were only extracted and principal components were analyzed on the basis of correlation matrix.

Stepwise Discriminant Analysis (SDA) of reflectance spectra

To further reduce the number of wavelengths and to find the most uncorrelated ones out of the total number of wavelengths obtained after performing factor analysis in RS, SDA was used. SDA is one of the most efficient statistical techniques used to discriminate between groups. In this work forward linear SDA was used. At each step, all variables are evaluated within the model. Those variables that fail to meet the set criterion to stay within the model are removed stepwise, whereas those that can contribute most to the discriminant function are retained within the model based on $F$ value and Wilks' Lambda $(L)$. The procedure stops when no further variables are removed (Zhang et al., 2014).

The Wilks' Lambda $(L)$, a measure of discrimination used in the selection of bands is given by (Green and Caroll, 1978).

$$
L=\frac{\left|S_{\text {effect }}\right|}{\left|S_{\text {effect }}\right|+\left|S_{\text {error }}\right|}
$$

Where, $S$ is a matrix which is also known as sum of squares and cross-products. It is a multivariate test of significance and ranges between 0 and 1 . The reflectance values were the quantities used for computing the $S$ matrix. The values of $L$ are indicative of separability or discriminatory power of spectral wavebands. The values close to 0 indicate that the group means are different and values close to lindicate that they are not different and 1 indicates all means are the same. When there is no further significant decrease in $L$ value the band selection process stops (Panigrahy et al., 2012). A combination of low $L$ and high $F$-value shows higher discrimination and a 0 value of $L$ indicated perfect separability.

\section{Calculation of spectral distance}

The quantification of spectral separability for the 91 pairs was done for discriminating between the mangrove species. The separability criterion used was the square of Jeffries-Matusita $(\mathrm{J}-\mathrm{M})$ distance. $\mathrm{J}-\mathrm{M}$ distance calculates the separability of a pair of probability distributions (Richards and Jia, 2006). It is calculated as

$$
J-M_{i j}=\sqrt{2\left(1-e^{-d}\right)}
$$

Where,

$$
\begin{aligned}
d=\frac{1}{8}\left(\mu_{i}-\mu_{j}\right)^{T} & \left(\frac{C_{i}+C_{j}}{2}\right)^{-1}\left(\mu_{i}-\mu_{j}\right) \\
& +\frac{1}{2} \ln \left(\frac{\left|\left(C_{i}+C_{j}\right) / 2\right|}{\sqrt{\left|C_{i}\right| \times\left|C_{j}\right|}}\right)
\end{aligned}
$$

$i$ and $j$ spectral responses of the two mangrove classes being compared

$C \quad$ covariance matrix of the spectral response

$\mu \quad$ mean vector of the spectral response

In natural logarithm function

$\mathrm{T}$ transposition function

$|C|$ determinant of $C$

The squared $\mathrm{J}-\mathrm{M}$ distance ranges between 0 and 2, where the maximum value 2 represents maximum separability (Richards and Jia, 2005). As recommended by Thomas et al. (2002) and also in view of the widespread practice in remote sensing, a threshold value of 1.90 were set to determine whether the spectral groups are separable or not.

\section{Results}

Reflectance spectra, derivative spectra and continuum removed spectra

Reflectance spectra (RS), their corresponding first derivative spectra (FDS), second derivative spectra (SDS) and continuum removed reflectance spectra (CRRS) [here after referred as 'four spectral forms' of canopies of 14 mangrove species belonging to 9 families are presented in Figure 3. 


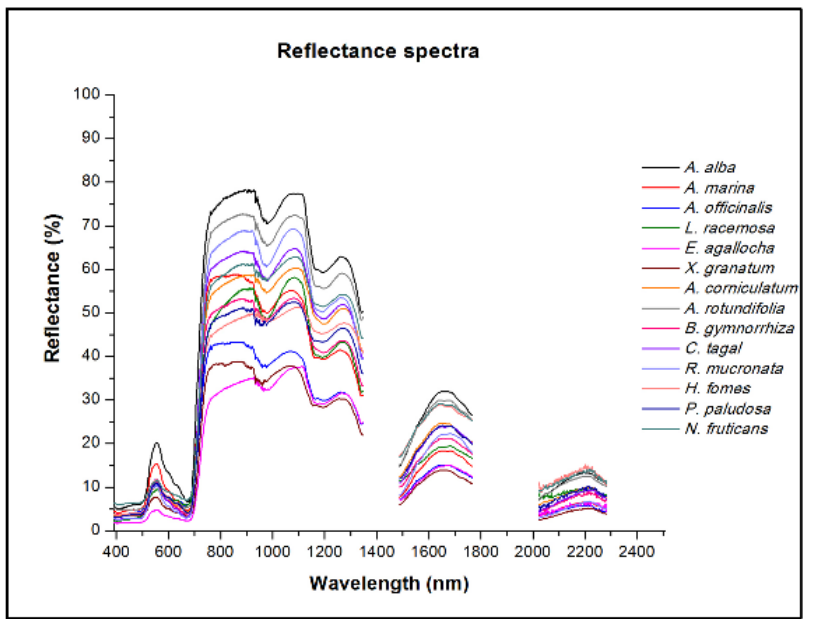

(a)

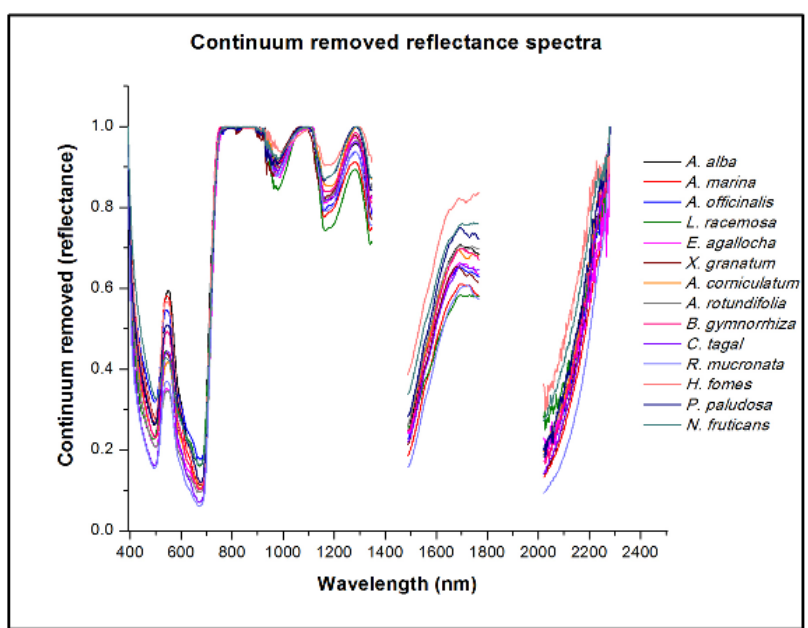

(d)

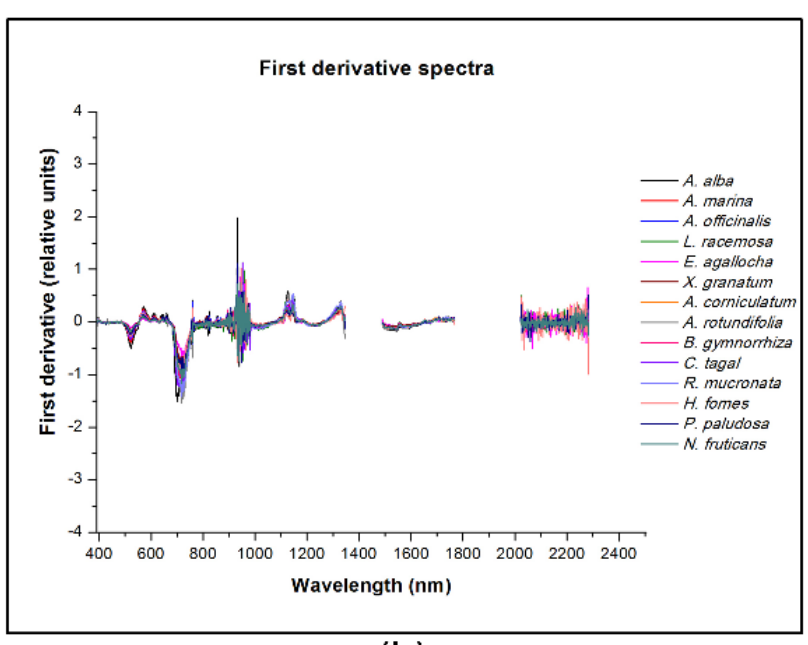

(b)

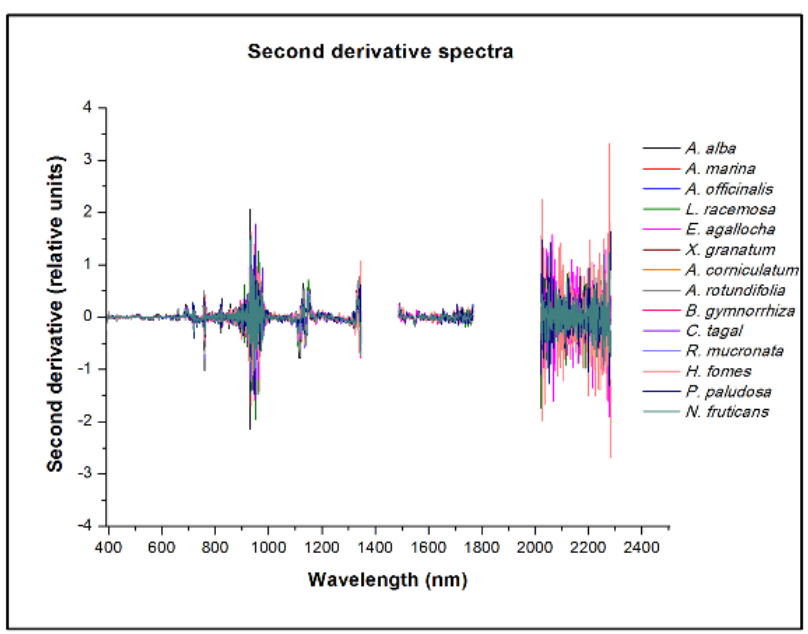

(c)

Figure 3 - Reflectance spectra, first derivative spectra, second derivative spectra and continuum removed reflectance spectra of the canopies of 14 mangrove species.

\section{Spectral separability analysis using statistical tests}

The wavelengths (bands) which were spectrally significant for each species pair were identified using Mann-Whitney U test at $95 \%$ and 99\% confidence levels. The results at 99\% confidence interval for RS, FDS, SDS and CRRS have been only discussed. The wavelength range (391-2281 nm) was divided into four major wavelength/ spectral regions (V - visible: 391$700 \mathrm{~nm}$; NIR - near infrared: 701-1500 nm (excluding 1350-1485); SWIR 1 - shortwave infrared 1: $1503-1766 \mathrm{~nm}$; SWIR 2 - shortwave infrared 2: 2022-2281 nm) for the ease of interpreting the results.

The pair-wise spectral separability for 91 species pairs in four spectral regions under four spectral forms have been represented in Table 2. Each value in the table represents the proportion in percentage of significant wavelengths for each spectral region out of the total number of wavelengths in that region for each of the species pair and spectral form. For RS form, there were nine species pairs which were separable in all spectral regions (shaded in green), 15 pairs separable in three spectral regions (shaded in blue) and 37 pairs separable in either two or a single spectral region (shade in red). In CRRS form there were three species pairs that were separable in all spectral regions (shaded in green), 11 pairs in three spectral regions (shaded in blue) and 33 pairs in either two/ one spectral region (shaded in red). It was observed that in CRRS form the spectral separability had decreased in the visible, NIR and SWIR 2 regions but increased in the SWIR 1 region in comparison to the RS form. Moreover, the separability had decreased in 42 pairs (shaded in orange), while increased in 24 pairs (shaded in grey) when RS was compared to CRRS. In FDS there was only one pair separable in three spectral regions (shaded in blue) and 18 pairs separable in either two/ one spectral region (shaded 
in red); besides, it was also noticed that the separability had increased in four and three pairs in the visible and SWIR 1 regions, respectively (depicted in red digits). Out of the 91 species pairs, 16 pairs recorded 0 to $<50 \%$ of separable bands in all spectral regions (shaded in yellow). Four species pairs recorded $>90 \%$ separable bands in RS form and in all spectral regions. A single pair (Rhizophora mucronata vs. Avicennia alba) recorded $>90 \%$ separable bands in three spectral regions of CRRS. The result represented in Figure 4 shows the number of species pairs which have $\geq 50 \%$ \& $<90 \%$ and $\geq$ $90 \%$ of separable bands in each of the spectral regions. Visible regions of RS, FDS and CRRS registered 17, 12 and 12 species pairs that had separable bands in the range $\geq 50 \%$ \& $<90 \%$, respectively and SWIR 2 of CRRS recorded the highest number of pairs (20). SWIR 1 of both RS and CRRS recorded the highest numbers of species pairs that had $\geq 90 \%$ of separable bands ( 32 and 28 in RS and CRRS, respectively). For FDS, bands were separable only in the range $\geq 50 \% \&<90 \%$. The pairwise spectral separability was less than $50 \%$ in the spectral regions in SDS form [Table 2 and Figure 4].

Table 3 represents the percentages of significant (separable) wavelengths under each spectral region out of the total number of significant bands for 91 species pairs and four spectral forms. In RS form the contributions of visible, NIR and SWIR 2 regions in pair-wise species separability were greater than $50 \%$ in 12 (shaded in yellow), 39 (shaded in blue) and eight pairs (shaded in green), respectively. Likewise, in CRRS the proportion of significant wavelengths under visible, NIR and SWIR 2 regions were greater than $50 \%$ in seven (yellow), 49 (blue) and one pair (green), correspondingly. The significant bands were highest in the visible and NIR regions of 16 (yellow) and 43 (blue) pairs, respectively for FDS and in the NIR region of 89 pairs (blue) for SDS. None of the bands were found to be separable in any of the spectral regions in 15 pairs in RS form (shaded in red) and six pairs in FDS form (shaded in purple). Figure 5 shows that the highest numbers of species pairs with separable wavelengths in the range of $\geq 50 \% \&<90 \%$ were obtained under the NIR regions in all the spectral forms. Similarly, the highest numbers of pairs with separable wavelengths $\geq 90 \%$ were observed in the NIR regions for RS and CRRS spectral forms. Thus, the contribution of NIR region in the spectral separability of mangrove species was higher than the visible and both the SWIR regions.

Scatter plots (Figure 6) show the location of spectrally significant (separable) bands at each wavelength location obtained from Mann-Whitney U test for the 91 species pairs in the four spectral forms at $99 \%$ confidence interval. In $\mathrm{RS}$ all bands were found to be significant (separable) in some species pairs or the other, excluding three pairs, namely pairs 58 to 60 i.e Ceriops decandra vs. Aegiceras corniculatum, $C$. decandra vs. Aegialitis rotundifolia and $C$. decandra vs. $R$. mucronata (Figure 6 a). In FDS, most of the locations of spectrally significant (separable) bands were found to be in the wavelength range of $493 \mathrm{~nm}$ to $966 \mathrm{~nm}$; the insignificant bands were 392, 2254, 2262 and 2269 nm (Figure 6 b). None of the wavelengths were found to be spectrally significant in six species pairs, viz. pairs 56 to 61 i.e. C. decandra vs. A. alba, C. decandra vs. A. marina, C. decandra vs. A. corniculatum, C. decandra vs. A. rotundifolia, $C$. decandra vs. $R$. mucronata and $C$. decandra vs. $X$. granatum for FDS (Figure $6 \mathrm{~b}$ ). In case of SDS most of the locations of spectrally significant bands were found to be in the range of 682-987 nm and 89 bands were found to be insignificant $(400,415,423,426,434,454-455,458$, $460,479,488-490,493-494,497,506,527,570$, $583,586-589,591-593,596,600,607-609,614$, 619, 627-629, 639, 641, 644, 648, 657, 667, 671, 675-676, 824, 1068, 1095, 1261, 1276, 1328, 1346, $1492,1503,1514,1521,1532,1543-1346,1564$, 1582, 1592-1599, 1614, 1621, 1645, 1683, 1721, $1746,1759,2038,2046,2057,2062,2070,2078-$ 2080, 2101, 2112, 2133, 2153-2158, 2199, 2207, 2234, 2254 and $2281 \mathrm{~nm}$ ) (Figure $6 \mathrm{c}$ ). For CRRS spectral form, the most frequent locations of spectrally separable bands were observed in the ranges of $1156-1335 \mathrm{~nm}, 1489-1766 \mathrm{~nm}$ and $2022-$ $2279 \mathrm{~nm}$; the insignificant wavelengths were 391, 853 and $2281 \mathrm{~nm}$ (Figure $6 \mathrm{~d}$ ).

Frequency plots (Figure 7) depict the number of species pairs separable in each wavelength (band) in the four spectral forms. A threshold value of $t=45$ is fixed (about $50 \%$ of the total number of species pairs -91) and those bands which have values more than 45 are plotted in black colour. Under RS the wavelength which were significant in more than 45 species pairs were 746, 758-761, 768-769, 771-774, 776-778, 780-782, 784-785 nm (total 19 wavelengths), for FDS the bands were 24 in number (509, 511, 512, 514-515, 518, 520-521, 523, 526527, 530, 532-533, 571, 574, 576-578, 580, 700, 713 $\mathrm{nm})$, under SDS only two bands (870 and $881 \mathrm{~nm}$ ), for CRRS a total of 61 bands $(961,964,966-968$, 970-971, 1007-1010, 1014, 1018, 1022, 1156, 1159, $1163,1167,1171,1175,1178,1182,1186,1190$, $1193,1197,1201,1205,1209,1212,1216,1220$, $1224,1227,1231,1235,1239,1242,1246,1250$, $1254,1257,1261,1265,1269,1272,1276,1280$, 1283, 1287, 1291, 1295, 1298, 1302, 1306, 1309, $1313,1317,1321,1324,1328,1332,1335 \mathrm{~nm})$. 
Table 2 - Results of Mann-Whitney U test showing spectral separability in two cases, viz. $\geq 50 \% \&<90 \%$ (depicted in bold and italics) and $\geq 90 \%$ (in bold) in each spectral region. Abbreviations of species as in Table 1. Other abbreviations as in the text. The digits in red and the shaded colours have been discussed in the text.

\begin{tabular}{|c|c|c|c|c|c|}
\hline Species & & & & & \\
\hline pair & $\mathrm{V}$ & NIR & SWIR 1 & SWIR 2 & $\mathrm{~V}$ \\
\hline Pp vs. Ea & 100.00 & 3.89 & 76.32 & 50.98 & 30.66 \\
\hline Pp vs. Hf & - & 1.20 & 25.00 & 97.06 & 2.36 \\
\hline Pp vs. Lr & - & - & - & - & 8.02 \\
\hline Pp vs. Bg & - & - & - & - & 8.02 \\
\hline $\mathrm{Pp}$ vs. $\mathrm{Cd}$ & - & - & - & - & 15.09 \\
\hline Pp vs. $\mathrm{Xg}$ & 32.08 & 3.59 & 100.00 & 99.02 & 21.23 \\
\hline Pp vs. Rm & 16.98 & - & - & 54.90 & 7.08 \\
\hline Pp vs. Ar & - & - & - & - & 8.96 \\
\hline Pp vs. Ac & - & - & - & - & 2.36 \\
\hline Pp vs. Am & 12.26 & 5.69 & - & 50.00 & 33.02 \\
\hline Pp vs. Aa & 50.47 & 99.40 & 100.00 & 75.49 & 48.58 \\
\hline Pp vs. Ao & - & - & - & 28.43 & 5.66 \\
\hline Pp vs. Nf & 47.17 & 1.20 & 17.11 & 97.06 & 4.72 \\
\hline Hf vs. Lr & 27.83 & 27.25 & 100.00 & 31.37 & 40.57 \\
\hline Hf vs. Bg & - & 2.10 & 100.00 & 100.00 & 21.23 \\
\hline Hf vs. Cd & 35.38 & 38.92 & - & 49.02 & 21.23 \\
\hline Hf vs. Xg & 52.36 & 82.04 & 100.00 & 100.00 & 53.30 \\
\hline Hf vs. Rm & 33.02 & 37.43 & 39.47 & 100.00 & 12.26 \\
\hline Hf vs. Ar & - & 47.90 & - & - & 24.06 \\
\hline Hf vs. Ac & - & 70.06 & 100.00 & 88.24 & 20.75 \\
\hline Hf vs. Am & 25.00 & 61.68 & 100.00 & 100.00 & 41.04 \\
\hline Hf vs. Aa & 70.28 & 98.80 & - & 0.98 & 48.58 \\
\hline Hf vs. Ao & - & 53.89 & 100.00 & 100.00 & 31.13 \\
\hline Hf vs. Nf & 69.34 & 72.75 & - & - & 46.23 \\
\hline Rm vs. Ar & - & - & - & - & 3.77 \\
\hline Rm vs. Ac & - & - & - & - & 3.30 \\
\hline Rm vs. Am & 82.08 & - & $\begin{array}{lll}- & - \\
-1 & \end{array}$ & - & 8.02 \\
\hline Rm vs. Aa & 100.00 & 69.76 & 100.00 & 100.00 & 14.15 \\
\hline Rm vs. Ao & & 88.32 & - & - & 26.42 \\
\hline Rm vs. Nf & 81.13 & 1.20 & - & 100.00 & 41.98 \\
\hline Cd vs. $\mathrm{Xg}$ & 18.87 & 100.00 & 100.00 & 88.24 & - \\
\hline Cd vs. Rm & - & - & - & - & - \\
\hline Cd vs. Ar & - & - & - & - & - \\
\hline Cd vs. Ac & - & - & - & - & - \\
\hline Cd vs. Am & 59.43 & - & - & - & - \\
\hline Cd vs. Aa & 100.00 & 0.90 & - & - & - \\
\hline Cd vs. Ao & - & 92.22 & - & - & 40.57 \\
\hline Cd vs. Nf & 66.51 & - & - & 30.39 & 45.28 \\
\hline Bg vs. Cd & - & - & - & 3.92 & 4.25 \\
\hline Bg vs. $\mathrm{Xg}$ & 17.92 & 100.00 & 100.00 & 87.25 & 45.28 \\
\hline Bg vs. Rm & - & - & - & - & 2.83 \\
\hline Bg vs. Ar & - & - & - & - & 12.26 \\
\hline Bg vs. Ac & - & 2.10 & - & - & 11.32 \\
\hline Bg vs. Am & 50.47 & 0.60 & - & - & 39.62 \\
\hline Bg vs. Aa & 83.96 & 100.00 & 100.00 & 100.00 & 48.11 \\
\hline Bg vs. Ao & - & 15.87 & - & - & 27.83 \\
\hline Bg vs. Nf & 72.64 & 2.10 & 100.00 & 100.00 & 37.74 \\
\hline Ar vs. Ac & - & - & - & - & 0.94 \\
\hline Ar vs. Am & - & - & - & - & 39.62 \\
\hline Ar vs. Aa & 13.21 & - & - & - & 49.06 \\
\hline Ar vs. Ao & - & 91.92 & 27.63 & - & 31.13 \\
\hline Ar vs. Nf & - & - & - & - & 14.62 \\
\hline Ac vs. Am & 18.87 & 21.86 & 100.00 & - & 40.57 \\
\hline Ac vs. Aa & 47.17 & 98.80 & 100.00 & 52.94 & 54.72 \\
\hline Ac vs. Ao & - & 93.41 & 97.37 & - & 3.30 \\
\hline Ac vs. $\mathrm{Nf}$ & 26.42 & 1.20 & - & 93.14 & 16.51 \\
\hline $\mathrm{Xg}$ vs. $\mathrm{Rm}$ & - & 96.41 & 19.74 & - & 30.66 \\
\hline Xg vs. Ar & - & 99.10 & 100.00 & 100.00 & 25.94 \\
\hline $\mathrm{Xg}$ vs. Ac & 0.94 & 100.00 & 100.00 & 100.00 & 30.19 \\
\hline $\mathrm{Xg}$ vs. Am & 66.51 & 98.80 & 32.89 & 23.53 & 61.32 \\
\hline Xg vs. Aa & 86.79 & 100.00 & 100.00 & 100.00 & 62.74 \\
\hline Xg vs. Ao & 11.79 & 2.40 & - & - & 29.25 \\
\hline Xg vs. Nf & 100.00 & 100.00 & 100.00 & 100.00 & 17.45 \\
\hline Ea vs. Hf & 100.00 & 100.00 & 100.00 & 100.00 & 58.49 \\
\hline Ea vs. Lr & 58.49 & 99.40 & 10.53 & - & 33.49 \\
\hline Ea vs. Bg & 89.15 & 60.78 & 1.32 & - & 55.66 \\
\hline Ea vs. Cd & 45.28 & 94.61 & 2.63 & - & 57.08 \\
\hline Ea vs. $\mathrm{Xg}$ & 38.68 & - & - & 3.92 & 9.43 \\
\hline Ea vs. Rm & 25.94 & 89.52 & - & - & 39.62 \\
\hline Ea vs. Ar & 23.11 & 99.70 & 100.00 & - & 35.85 \\
\hline Ea vs. Ac & 73.11 & 100.00 & 100.00 & - & 39.62 \\
\hline Ea vs. Am & 100.00 & 82.63 & - & - & 65.09 \\
\hline Ea vs. Aa & 100.00 & 100.00 & 100.00 & 96.08 & 66.04 \\
\hline Ea vs. Ao & 98.11 & 12.28 & - & - & 35.85 \\
\hline Ea vs. $\mathrm{Nf}$ & 100.00 & 100.00 & 100.00 & 100.00 & 2.83 \\
\hline Lr vs. Bg & 35.85 & - & - & - & 49.53 \\
\hline Lr vs. Cd & - & 15.57 & - & - & 44.81 \\
\hline Lr vs. Xg & 13.68 & 100.00 & 100.00 & 100.00 & 28.30 \\
\hline Lr vs. $\mathrm{Rm}$ & - & 12.28 & - & 46.08 & 20.28 \\
\hline Lr vs. Ar & - & 27.25 & - & - & 17.45 \\
\hline Lr vs. Ac & - & 23.05 & - & - & 20.75 \\
\hline Lr vs. Am & 44.34 & 44.91 & - & - & 47.64 \\
\hline Lr vs. Aa & 70.28 & 98.80 & 94.74 & - & 55.19 \\
\hline Lr vs. Ao & 1.89 & 87.43 & 25.00 & - & 13.68 \\
\hline Lr vs. Nf & 48.11 & 52.10 & 100.00 & - & 15.57 \\
\hline Ao vs. Nf & 22.64 & 93.11 & 100.00 & 100.00 & 16.51 \\
\hline Am vs. Aa & 36.79 & 100.00 & 100.00 & 100.00 & 17.92 \\
\hline Am vs. Ao & 6.60 & 91.62 & - & - & 46.70 \\
\hline Am vs. Nf & 24.06 & 29.34 & 100.00 & 100.00 & 56.13 \\
\hline Aa vs. Ao & 31.60 & 98.80 & 100.00 & 88.24 & 58.96 \\
\hline Aa vs. Nf & 40.09 & 64.97 & - & - & 31.13 \\
\hline
\end{tabular}




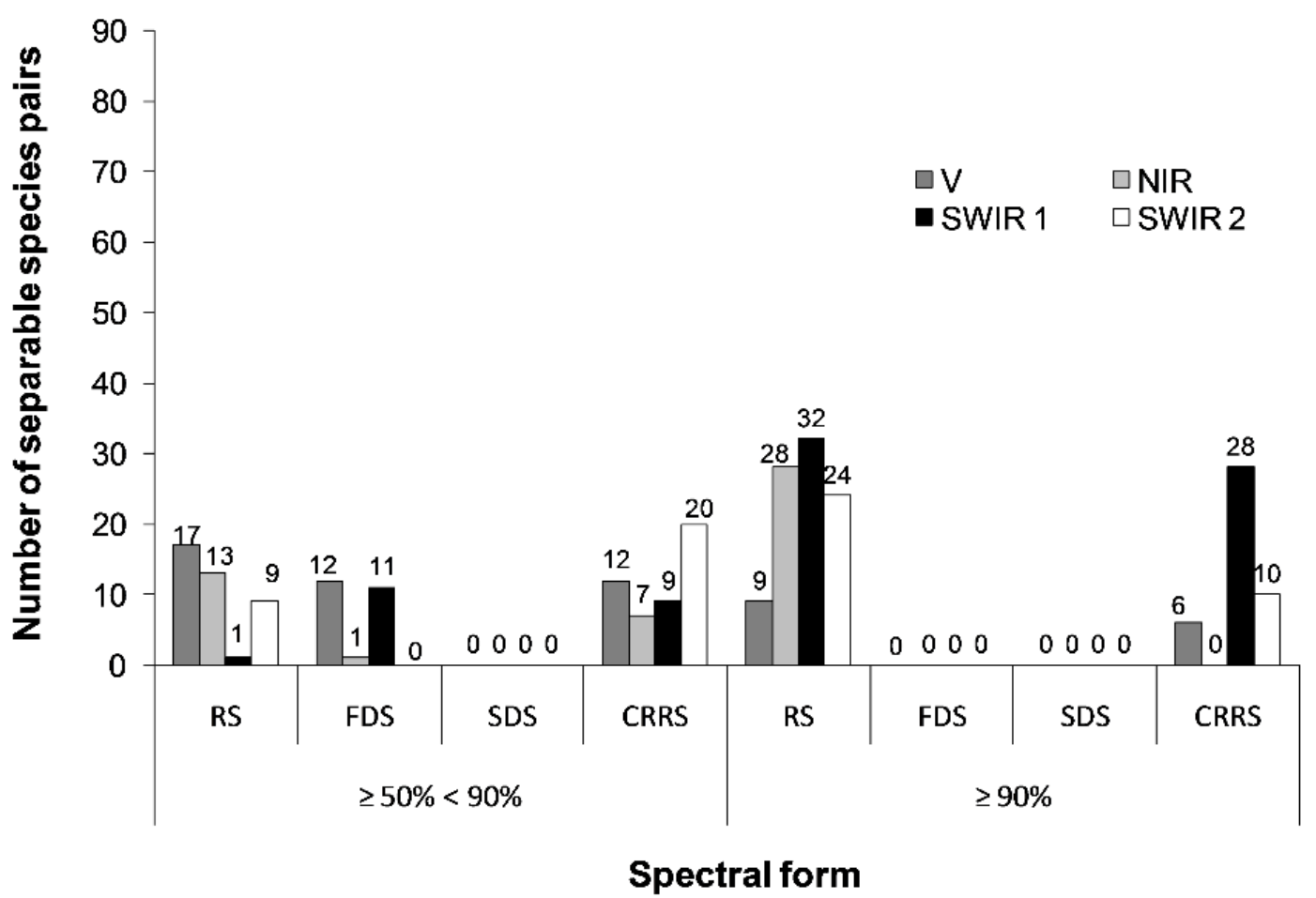

Figure 4 - Number of species pairs with $\geq 50 \% \&<90 \%$ and $\geq 90 \%$ of separable bands in each spectral region of the four spectral forms obtained from Mann-Whitney U test. Abbreviations as in the text.

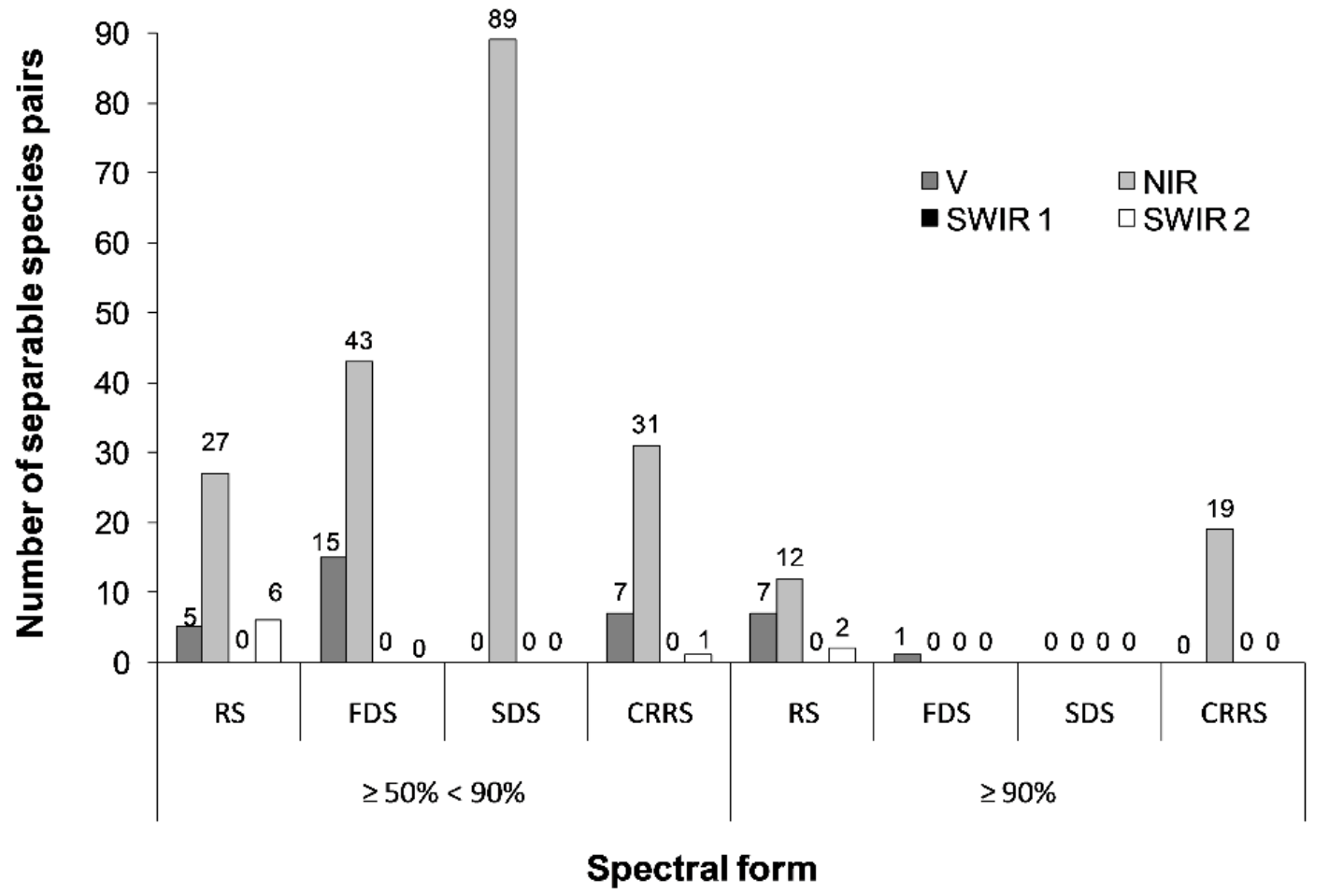

Figure 5 - Number of species pairs with $\geq 50 \%$ \& $<90 \%$ and $\geq 90 \%$ of significant wavelengths under each spectral region out of the total number of significant bands for each of the 91 species pair and four spectral forms. Abbreviations as in the text. 
Table 3 - Results of Mann-Whitney U test showing the percentages of significant wavelengths in two cases, viz. $\geq 50 \%$ \& $<90 \%$ (depicted in bold and italics) and $\geq 90 \%$ (in bold) in each spectral region. Abbreviations of species as in Table 1. Other abbreviations as in the text. The digits in red and the shaded colours have been discussed in the text.

\begin{tabular}{|c|c|c|c|c|c|}
\hline Snecies pair & & & & & \\
\hline Species pair & $\mathrm{V}$ & NIR & SWIR 1 & SWIR 2 & $\mathrm{~V}$ \\
\hline Pp vs. Ea & 63.28 & 3.88 & 17.31 & 15.52 & 57.02 \\
\hline $\mathrm{Pp}$ vs. Hf & - & 3.28 & 15.57 & 81.15 & - \\
\hline $\mathrm{Pp}$ vs. $\mathrm{Lr}$ & - & - & - & - & 12.50 \\
\hline Pp vs. $B g$ & - & - & - & - & 33.33 \\
\hline Pp vs. Cd & - & - & - & - & 38.55 \\
\hline Pp vs. Xg & 26.46 & 4.67 & 29.57 & 39.30 & 36.89 \\
\hline Pp vs. Rm & 39.13 & - & - & 60.87 & 15.31 \\
\hline Pp vs. Ar & - & - & - & - & 25.68 \\
\hline Pp vs. Ac & - & - & - & - & 8.47 \\
\hline Pp vs. Am & 27.08 & 19.79 & - & 53.13 & 47.95 \\
\hline Pp vs. Aa & 18.07 & 56.08 & 12.84 & 13.01 & 46.40 \\
\hline Pp vs. Ao & - & - & - & 100.00 & 13.95 \\
\hline Pp vs. Nf & 46.30 & 1.85 & 6.02 & 45.83 & 19.61 \\
\hline Hf vs. Lr & 22.87 & 35.27 & 29.46 & 12.40 & 37.07 \\
\hline Hf vs. Bg & - & 3.78 & 41.08 & 55.14 & 29.61 \\
\hline Hf vs. Cd & 29.41 & 50.98 & - & 19.61 & 23.81 \\
\hline Hf vs. $\mathrm{Xg}$ & 19.72 & 48.67 & 13.50 & 18.12 & 42.32 \\
\hline Hf vs. Rm & 21.41 & 38.23 & 9.17 & 31.19 & 13.83 \\
\hline Hf vs. Ar & - & 100.00 & - & - & 25.00 \\
\hline Hf vs. Ac & - & 58.50 & 19.00 & 22.50 & 29.14 \\
\hline Hf vs. Am & 12.13 & 47.14 & 17.39 & 23.34 & 32.95 \\
\hline Hf vs. Aa & 31.04 & 68.75 & - & 0.21 & 40.23 \\
\hline Hf vs. Ao & - & 50.28 & 21.23 & 28.49 & 30.28 \\
\hline Hf vs. Nf & 37.69 & 62.31 & - & - & 48.51 \\
\hline Rm vs. Ar & - & - & - & - & 11.43 \\
\hline Rm vs. Ac & - & - & - & - & 7.37 \\
\hline Rm vs. Am & 100.00 & - & - & - & 39.53 \\
\hline Rm vs. Aa & 34.03 & 37.40 & 12.20 & 16.37 & 40.00 \\
\hline Rm vs. Ao & - & 100.00 & - & - & 53.33 \\
\hline Rm vs. Nf & 61.87 & 1.44 & - & 36.69 & 68.99 \\
\hline Cd vs. $\mathrm{Xg}$ & 7.41 & 61.85 & 14.07 & 16.67 & - \\
\hline $\mathrm{Cd}$ vs. $\mathrm{Rm}$ & - & - & - & - & - \\
\hline Cd vs. $\mathrm{Ar}$ & - & - & - & - & - \\
\hline Cd vs. Ac & - & - & - & - & - \\
\hline $\mathrm{Cd}$ vs. Am & 100.00 & - & - & - & - \\
\hline Cd vs. Aa & 98.60 & 1.40 & - & - & - \\
\hline Cd vs. Ao & - & 100.00 & - & - & 36.75 \\
\hline $\mathrm{Cd}$ vs. $\mathrm{Nf}$ & 81.98 & - & - & 18.02 & 60.76 \\
\hline Bg vs. $\mathrm{Cd}$ & - & - & - & 100.00 & 15.52 \\
\hline Bg vs. $\mathrm{Xg}$ & 7.08 & 62.20 & 14.15 & 16.57 & 40.68 \\
\hline Bg vs. Rm & - & - & - & - & 4.65 \\
\hline Bg vs. Ar & - & - & - & - & 26.53 \\
\hline Bg vs. Ac & - & 100.00 & - & - & 28.24 \\
\hline Bg vs. Am & 98.17 & 1.83 & - & - & 52.83 \\
\hline Bg vs. Aa & 25.80 & 48.41 & 11.01 & 14.78 & 42.32 \\
\hline Bg vs. Ao & - & 100.00 & - & - & 32.78 \\
\hline Bg vs. $\mathrm{Nf}$ & 45.43 & 2.06 & 22.42 & 30.09 & 55.94 \\
\hline Ar vs. Ac & - & - & - & - & 4.76 \\
\hline Ar vs. Am & - & - & - & - & 52.50 \\
\hline Ar vs. Aa & 100.00 & - & - & - & 67.97 \\
\hline Ar vs. Ao & - & 93.60 & 6.40 & - & 30.56 \\
\hline Ar vs. Nf & - & - & - & - & 36.90 \\
\hline Ac vs. Am & 21.16 & 38.62 & 40.21 & - & 43.65 \\
\hline Ac vs. Aa & 17.86 & 58.93 & 13.57 & 9.64 & 51.10 \\
\hline Ac vs. Ao & - & 80.83 & 19.17 & - & 4.29 \\
\hline Ac vs. Nf & 36.13 & 2.58 & - & 61.29 & 34.31 \\
\hline $\mathrm{Xg}$ vs. $\mathrm{Rm}$ & - & 95.55 & 4.45 & - & 22.73 \\
\hline $\mathrm{Xg}$ vs. $\mathrm{Ar}$ & - & 65.03 & 14.93 & 20.04 & 23.31 \\
\hline $\mathrm{Xg}$ vs. Ac & 0.39 & 64.98 & 14.79 & 19.84 & 27.00 \\
\hline Xg vs. Am & 27.12 & 63.46 & 4.81 & 4.62 & 49.81 \\
\hline $\mathrm{Xg}$ vs. $\mathrm{Aa}$ & 26.44 & 47.99 & 10.92 & 14.66 & 36.84 \\
\hline Xg vs. Ao & 75.76 & 24.24 & - & - & 52.10 \\
\hline $\mathrm{Xg}$ vs. $\mathrm{Nf}$ & 29.28 & 46.13 & 10.50 & 14.09 & 17.13 \\
\hline Ea vs. $\mathrm{Hf}$ & 29.28 & 46.13 & 10.50 & 14.09 & 61.39 \\
\hline Ea vs. $\mathrm{Lr}$ & 26.72 & 71.55 & 1.72 & - & 34.63 \\
\hline Ea vs. Bg & 48.09 & 51.65 & 0.25 & - & 57.84 \\
\hline Ea vs. Cd & 23.19 & 76.33 & 0.48 & - & 54.26 \\
\hline Ea vs. $\mathrm{Xg}$ & 95.35 & - & - & 4.65 & 13.33 \\
\hline Ea vs. Rm & 15.54 & 84.46 & - & - & 100.00 \\
\hline Ea vs. Ar & 10.70 & 72.71 & 16.59 & - & 38.78 \\
\hline Ea vs. Ac & 27.43 & 59.12 & 13.45 & - & 46.67 \\
\hline Ea vs. Am & 43.44 & 56.56 & & - & 49.46 \\
\hline Ea vs. Aa & 29.44 & 46.39 & 10.56 & 13.61 & 46.20 \\
\hline Ea vs. Ao & 83.53 & 16.47 & - & - & 44.97 \\
\hline Ea vs. Nf & 29.28 & 46.13 & 10.50 & 14.09 & 1.32 \\
\hline Lr vs. Bg & 100.00 & - & - & - & 62.87 \\
\hline Lr vs. Cd & - & 100.00 & - & - & 45.89 \\
\hline Lr vs. $\mathrm{Xg}$ & 5.36 & 61.74 & 14.05 & 18.85 & 24.69 \\
\hline Lr vs. $\mathrm{Rm}$ & - & 46.59 & - & 53.41 & 20.87 \\
\hline Lr vs. Ar & - & 100.00 & - & - & 19.79 \\
\hline Lr vs. Ac & - & 100.00 & - & - & 23.53 \\
\hline Lr vs. Am & 38.52 & 61.48 & - & - & 37.55 \\
\hline Lr vs. Aa & 27.04 & 59.89 & 13.07 & - & 41.05 \\
\hline Lr vs. Ao & 1.27 & 92.70 & 6.03 & - & 14.95 \\
\hline Lr vs. Nf & 28.98 & 49.43 & 21.59 & - & 17.28 \\
\hline Ao vs. Nf & 8.94 & 57.91 & 14.15 & 18.99 & 29.17 \\
\hline Am vs. Aa & 13.22 & 56.61 & 12.88 & 17.29 & 19.79 \\
\hline Am vs. Ao & 4.38 & 95.63 & - & - & 45.00 \\
\hline Am vs. Nf & 15.60 & 29.97 & 23.24 & 31.19 & 53.13 \\
\hline Aa vs. Ao & 11.90 & 58.61 & 13.50 & 15.99 & 42.23 \\
\hline Aa vs. Nf & 28.15 & 71.85 & - & - & 35.68 \\
\hline
\end{tabular}




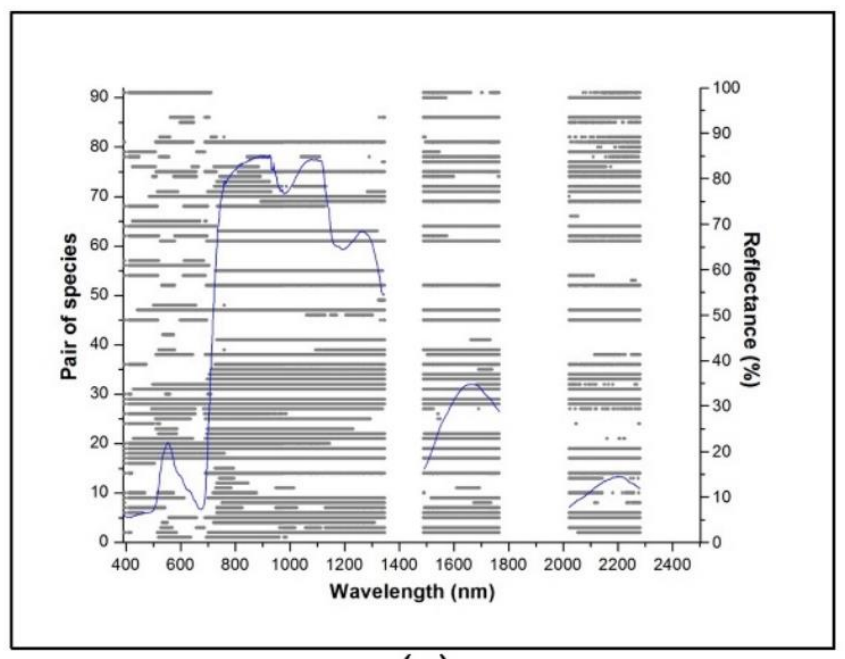

(a)

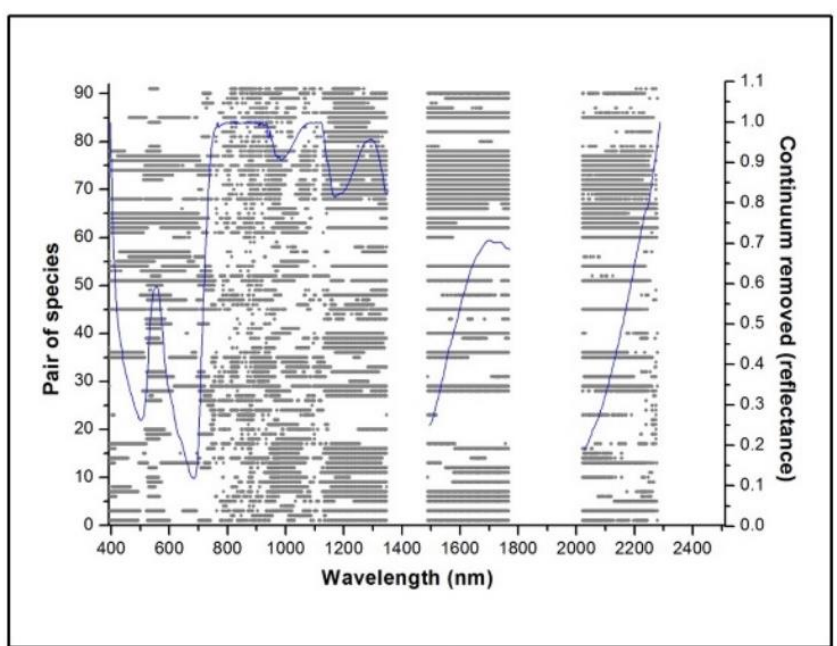

(d)

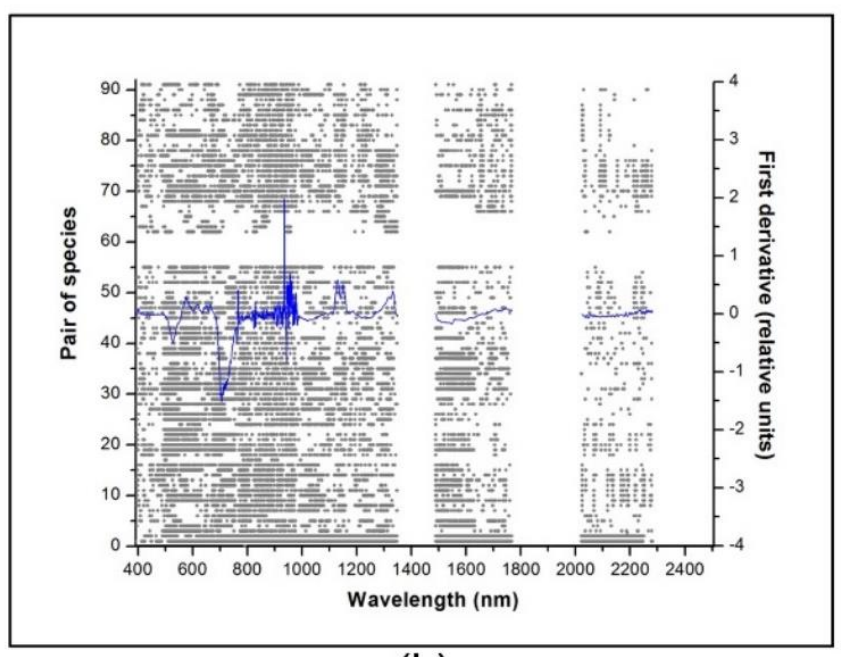

(b)

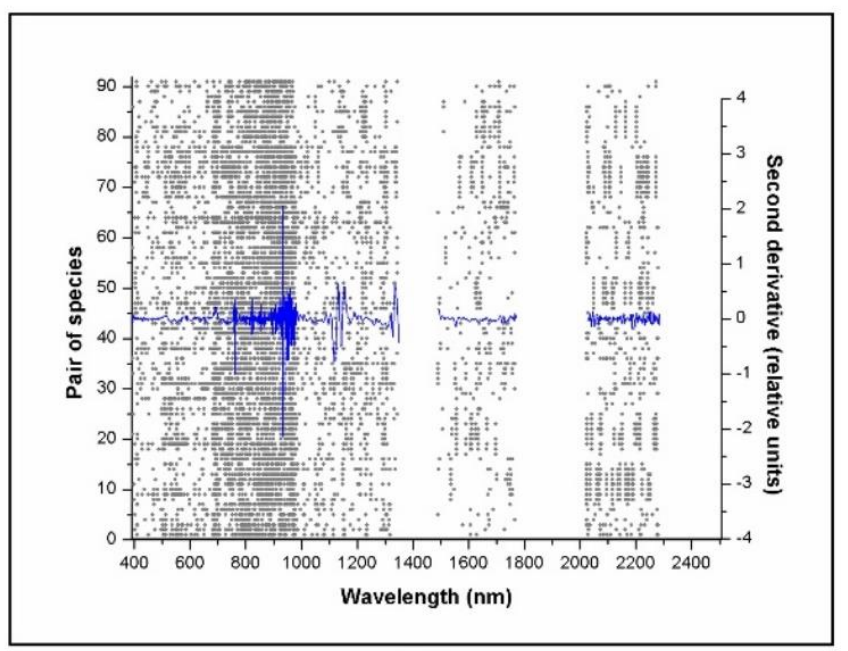

(c)

Figure 6 - Scatter plots depicting the location of spectrally significant (separable) bands at each wavelength location obtained from Mann-Whitney $U$ test for 91 species pairs in the four spectral forms at $99 \%$ confidence interval. The average spectrum of Avicennia alba in each spectral form is plotted in the respective scatter plots. 1 Aa vs. Nf, 2 - Aa vs. Ao, 3 - Am vs. Nf, 4 - Am vs. Ao, 5 - Am vs. Aa, 6 - Ao vs. Nf, 7 - Lr vs. Nf, 8 - Lr vs. Ao, 9 - Lr vs. Aa, 10 - Lr vs. Am, 11 - Lr vs. Ac, 12 - Lr vs. Ar, 13 - Lr vs. Rm, 14 - Lr vs. Xg, 15 - Lr vs. Cd, 16 Lr vs. Bg, 17 - Ea vs. Nf, 18 - Ea vs. Ao, 19 - Ea vs. Aa, 20 - Ea vs. Am, 21 - Ea vs. Ac, 22 - Ea vs. Ar, 23 - Ea vs. Rm, 24 - Ea vs. Xg, 25 - Ea vs. Cd, 26 - Ea vs. Bg, 27 - Ea vs. Lr, 28 - Ea vs. Hf, 29 - Xg vs. Nf, 30 - Xg vs. Ao, 31 - Xg vs. Aa, 32 - Xg vs. Am, 33 - Xg vs. Ac, 34 - Xg vs. Ar, 35 - Xg vs. Rm, 36 - Ac vs. Nf, 37 - Ac vs. Ao, 38 - Ac vs. Aa, 39 - Ac vs. Am, 40 - Ar vs. Nf, 41 - Ar vs. Ao, 42 - Ar vs. Aa, 43 - Ar vs. Am, 44 - Ar vs. Ac, $45-\mathrm{Bg}$ vs. Nf, $46-\mathrm{Bg}$ vs. Ao, $47-\mathrm{Bg}$ vs. Aa, $48-\mathrm{Bg}$ vs. Am, $49-\mathrm{Bg}$ vs. Ac, $50-\mathrm{Bg}$ vs. Ar, $51-\mathrm{Bg}$ vs. Rm, $52-$ Bg vs. Xg, 53 - Bg vs. Cd, $54-$ Cd vs. Nf, $55-$ Cd vs. Ao, $56-$ Cd vs. Aa, $57-\mathrm{Cd}$ vs. Am, $58-$ Cd vs. Ac, 59 - Cd vs. Ar, 60 - Cd vs. Rm, 61 - Cd vs. Xg, 62 - Rm vs. Nf, $63-$ Rm vs. Ao, $64-$ Rm vs. Aa, $65-$ Rm vs. Am, 66 - Rm vs. Ac, 67 - Rm vs. Ar, 68 - Hf vs. Nf, 69 - Hf vs. Ao, 70 - Hf vs. Aa, 71 - Hf vs. Am, 72 - Hf vs. Ac, 73 - Hf vs. Ar, 74 - Hf vs. Rm, 75 - Hf vs. Xg, 76 - Hf vs. Cd, 77 - Hf vs. Bg, 78 - Hf vs. Lr, 79 - Pp vs. Nf, 80 - Pp vs. Ao, 81 - Pp vs. Aa, 82 - Pp vs. Am, 83 - Pp vs. Ac, 84 - Pp vs. Ar, 85 - Pp vs. Rm, 86 - Pp vs. Xg, 87 - Pp vs. Cd, 88 - Pp vs. Bg, 89 - Pp vs. Lr, 90 - Pp vs. Hf, 91 - Pp vs. Ea. Abbreviations of species as in Table 1. 


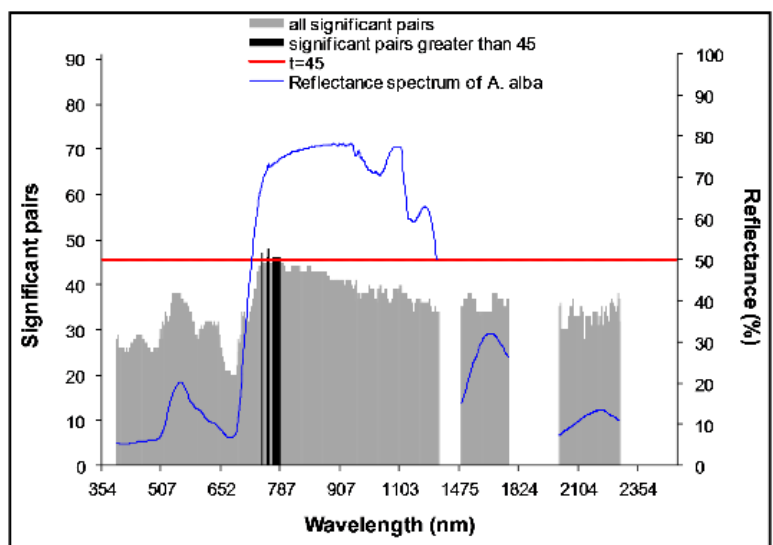

(a)

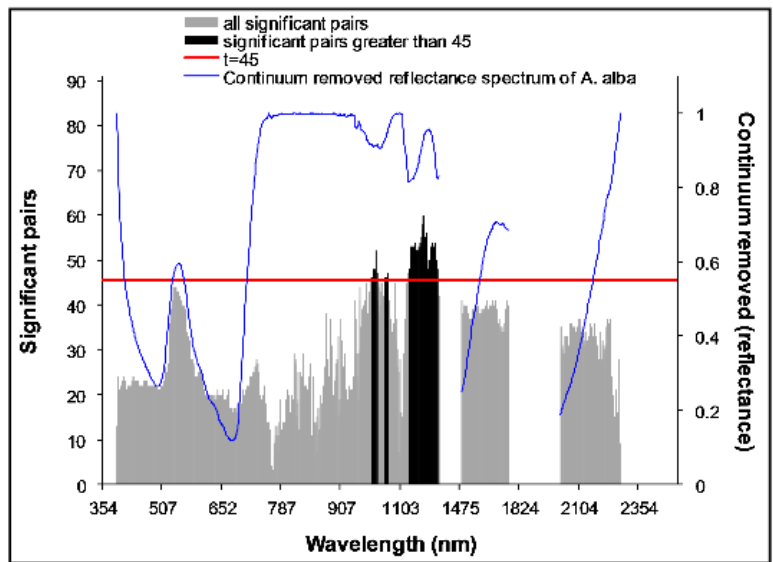

(d)

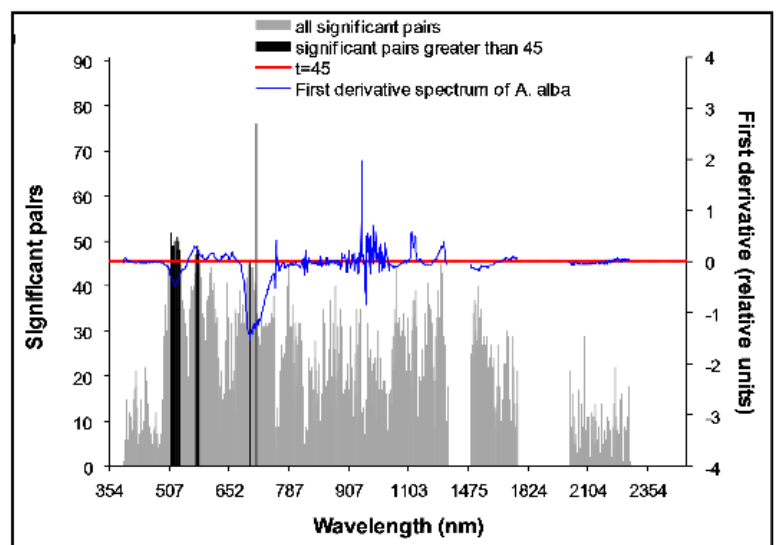

(b)

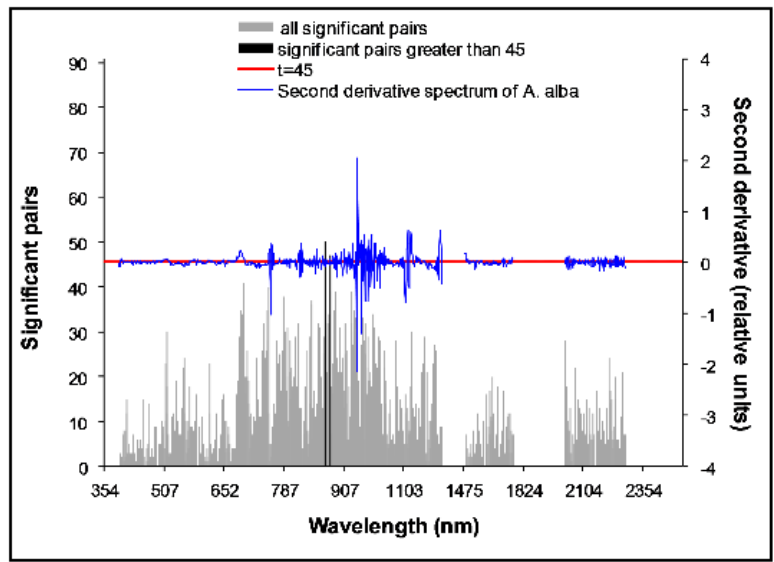

(c)

Figure 7 - Frequency plots depicting the number of statistically significant pairs at each wavelength location obtained from Mann-Whitney U test for 91 species pairs in the four spectral forms at $99 \%$ confidence interval. The average spectrum of Avicennia alba in each spectral form is plotted in the respective plots.

\section{Feature reduction analysis}

From the outcomes of the statistical tests (Mann-Whitney U tests) on the four spectral forms, bands were selected and used for further feature reduction using factor analysis. This was followed by stepwise discriminant analysis of RS and calculation of $\mathrm{J}-\mathrm{M}$ distance.

\section{Factor analysis}

Factor analysis was chosen for further band reduction analysis to select most uncorrelated bands from the input bands using PCA and Varimax method with Kaiser Normalization for rotation of the components. As per the factor loadings (correlation coefficients) the first $6,49,56$ and 20 factors/ components showed the maximum cumulative percentages of variance in RS, FDS, SDS and CRRS, respectively (Appendix 1) and these were selected for further analysis. From each component, bands were selected based on their coefficient value. About 200 bands each were selected in RS, FDS and CRRS and 187 bands in SDS (Appendix 1). The bands (wavelengths) which were redundant within the selected factors/ components were ignored. The location of the wavelengths selected is given in Figure 8 and represented using red coloured symbols.

In RS only bands in the NIR region got selected. Bands in the visible, NIR and SWIR 1 regions got selected in FDS. Some common bands of the NIR region which got selected in both the derivative spectra (FDS and SDS) and CRRS spectral form were 1152, 1156, 1159 and $1212 \mathrm{~nm}$. In addition to SWIR 1 bands, some of the SWIR 2 bands also got selected in SDS. Moreover, in the derivative spectra (both FDS and SDS) few bands of the green region (around $550 \mathrm{~nm}$ ) also got selected. Furthermore, in the derivative spectra and CRRS, few of the bands of the red absorbance region (around $650 \mathrm{~nm}$ ) and some of the bands of the red edge region $(680-720 \mathrm{~nm})$ also got chosen. The common selected 
SWIR 1 bands of SDS and CRRS were 1652 and $1694 \mathrm{~nm}$. Out of the four spectral forms, SDS was the only spectral form in which bands got selected from all the four spectral regions.

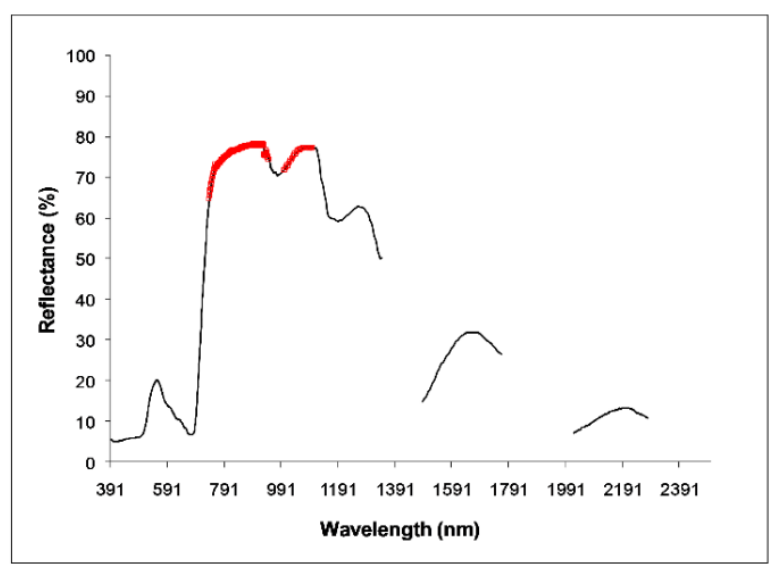

(a)

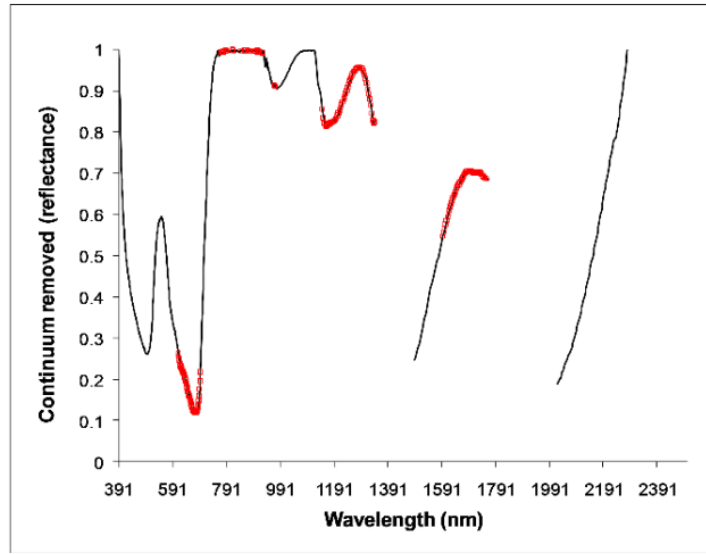

(d)

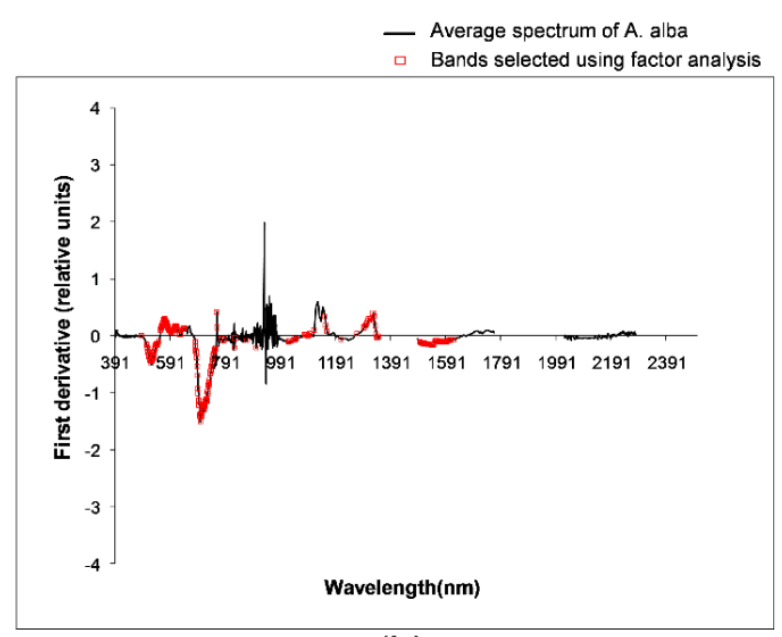

(b)

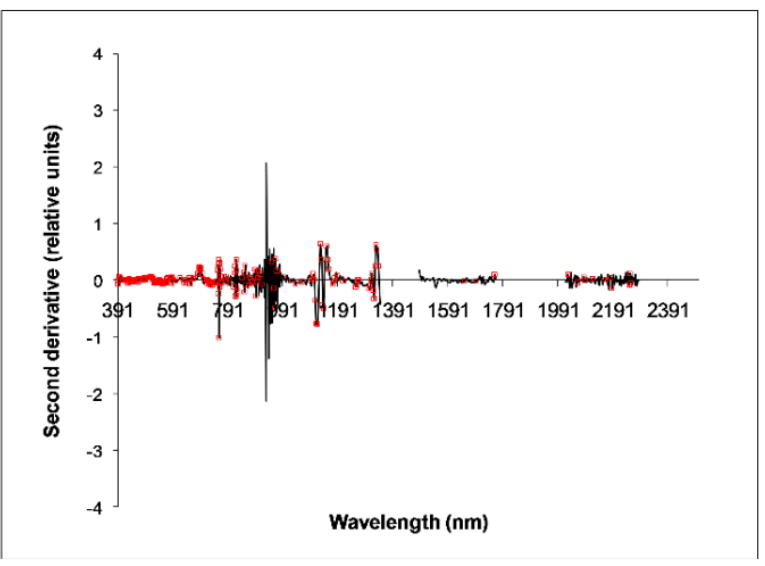

(c)

Figure 8 - Bands selected using factor analysis in four spectral forms.

Optimal wavelength selection using SDA

In order to further reduce the number of bands and to select the most optimal bands for quantitative analysis of spectral separability, SDA was performed and results were obtained for RS form. The selection of optimal bands depends on input data, the maximum $F$ and the minimum $L$ values involved in each step of the forward SDA methodology. The number of bands selected was 5 (Appendix 2) and the wavelength locations are represented in blue lines in Figure 9.

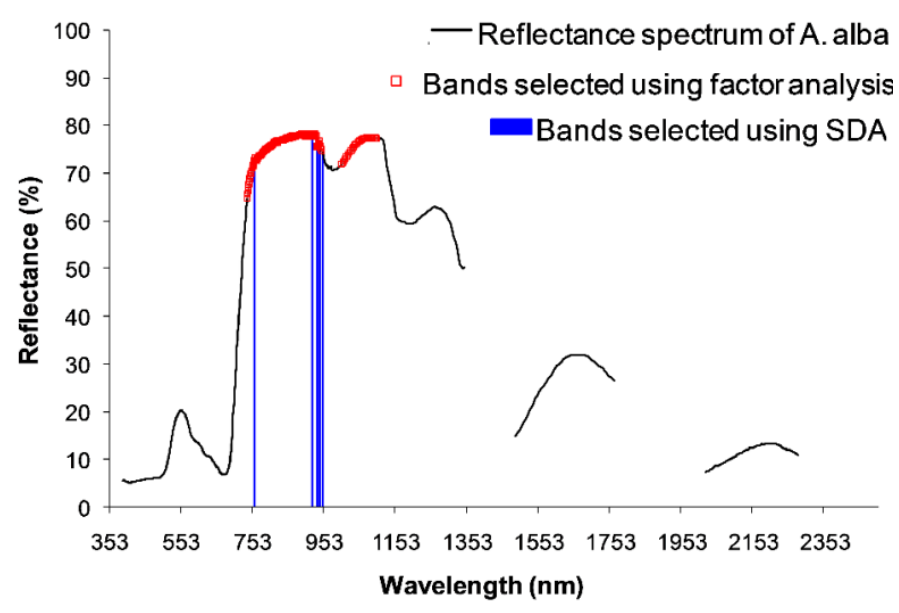


Figure 9 - Bands selected using feature reduction method, viz. factor analysis (represented by red symbols) and step-wise discriminant analysis (represented by blue lines).

Calculation of spectral distance

A threshold of 1.90 was fixed for the square of $\mathrm{J}-\mathrm{M}$ distance to assess the separability among each species pair. The increase in value towards 2.00 represented that there was increase in separability between species (Figure 10). In 64 species pairs the value was two. Twenty species pairs showed lesser spectral separability in comparison to the remaining pairs.

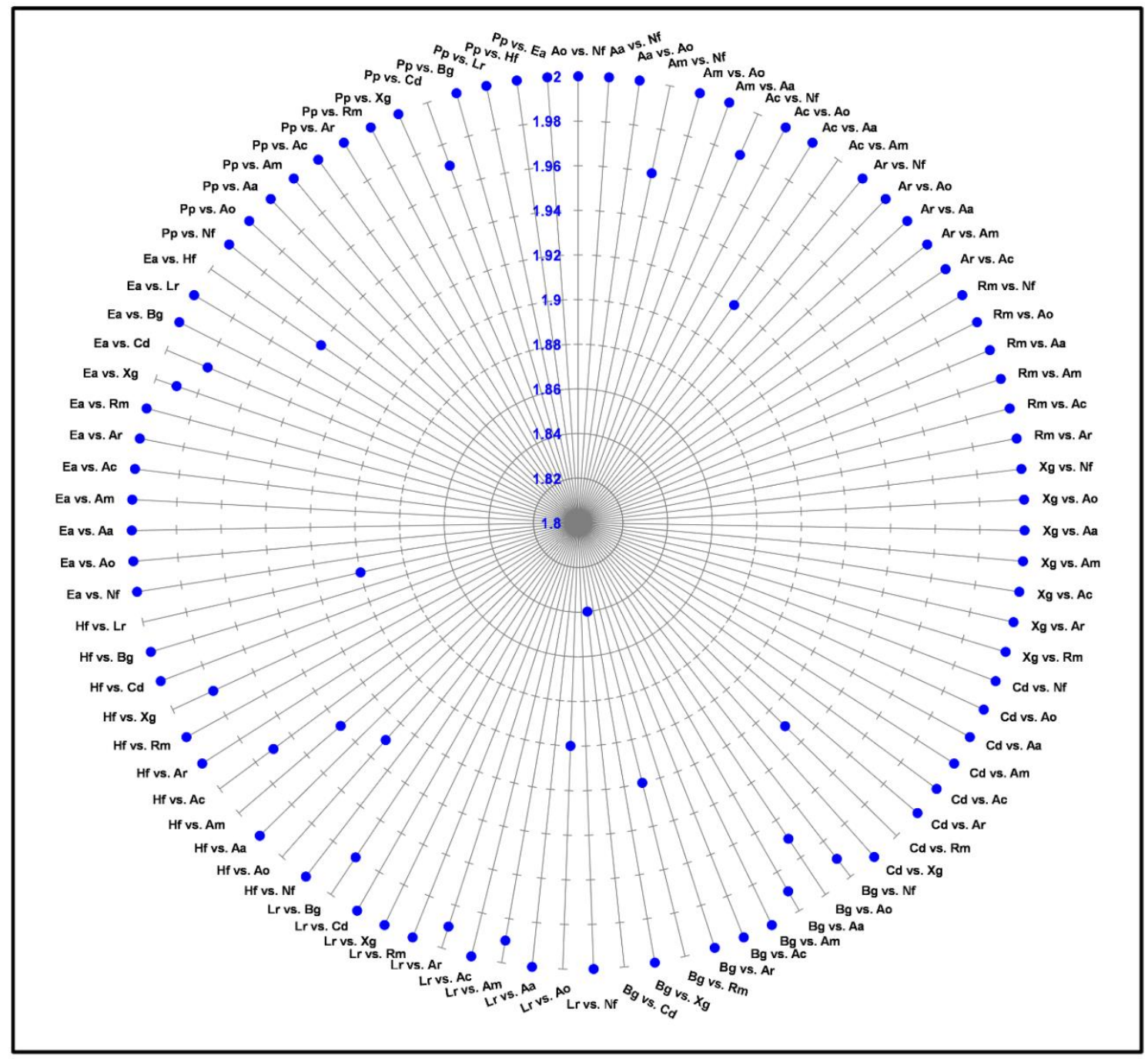

Figure 10 - Graphical representation of the squares of Jeffries-Matusita distances for 91 species pairs. Abbreviations of species as in Table 1.

\section{Discussion}

The broad shape of the reflectance spectra was found to be similar in all the 14 species of mangroves belonging to nine families. This revealed the similar biochemical and anatomical composition of vegetation across the species. The leaf biochemical information is transmitted virtually unchanged from the leaf to the canopy in near-infrared wavelengths (Asner, 1998). There is also multiple scattering within the leaves and canopy that causes broadening of absorption features, and reflectance from absorption features tend towards an asymptote when saturation is reached (Curran, 1989). However, as pigment concentrations, biochemical contents, leaf characteristics and canopy structure vary between species, so does absorption and reflectance (Asner, 1998; Martin et al., 1998). Moreover, it is an established fact that canopy spectra remain sensitive to variations in leaf biochemical content, leaf area index, canopy closure, canopy architecture, under storey reflectance, leaf anatomy and foliar water 
content (Dawson and Curran, 1998; Kumar et al., 2001). Therefore, generalizations cannot be made across different vegetation types as a whole and mangrove species per se (Knapp and Carter, 1998). Thus, there arises the need for transforming the field reflectance data into different spectral forms and using different statistical approaches for spectral discrimination of the species.

Since non-parametric statistical analysis is more flexible than parametric approach and depends on ordinals of values in place of the actual values which make the analysis easier (Prasad and Gnanappazham, 2016) hence field canopy spectral data of the 14 species were analyzed statistically using non-parametric approach (Mann-Whitney U test). Besides, non-parametric test makes fewer assumptions than parametric test (Manevski et al., 2011). The Mann-Whitney $U$ test showed that all the 14 mangrove species were spectrally distinguishable in several wavelengths, though the separability varied with the spectral forms and the spectral regions. On one hand the pair-wise spectral separability of 91 pairs (Table 2 and Figure 4) revealed that the RS form alone was suitable for separability analysis in four pairs of mangrove species, viz. $X$. granatum vs. N. fruticans, Excoecaria agallocha vs. Heritiera fomes, E. agallocha vs. A. alba and E. agallocha vs. $N$. fruticans, while on the other hand it established the unsuitability of RS form in spectral discrimination of 15 pairs. In addition, 16 pairs were least separable or inseparable in three or all spectral regions and at least one spectral form. In CRRS, the separability had decreased in the visible, NIR and SWIR 2 regions but increased in the SWIR 1 region in comparison to the RS form. Again, in comparison to RS the overall separability increased in CRRS for 24 pairs (Table 2). The percentages of significant (separable) wavelengths under each spectral region (Table 3 and Figure 5) showed that the contribution of NIR region in the spectral separability of species was higher than the other regions. Moreover, the scatter plots (Figure 6) revealed that the numbers of significant wavelengths were of the order RS> CRRS > FDS> SDS; additionally, it may be concluded that FDS is unsuitable for discrimination of six species pairs, i.e. C. decandra vs. A. alba, A. marina, A. corniculatum, A. rotundifolia, $R$. mucronata and $X$. granatum. Furthermore, the frequency plots (Figure 7) showed that in RS form more numbers of species pairs were separable in the visible (535-552 nm), NIR (728-1144 nm) and SWIR 1 (1532-1543 nm and 1677-1694 nm) regions than the remaining portions of the spectra. Likewise, in FDS more numbers of species pairs were separable in the visible (503-537 $\mathrm{nm}, 561-587 \mathrm{~nm}$ and 603$614 \mathrm{~nm})$, red edge $(689-713 \mathrm{~nm})$ and NIR (1026$1030 \mathrm{~nm}$ ) regions and for CRRS more numbers of species pairs were separable in the visible region (529-564 nm), NIR (941-1034 nm, 1064-1068 nm and 1140-1347 nm) and SWIR 1 (1489-1694 nm and 1746-1766 nm) regions. Thus, in both RS and CRRS quite a number of species pairs were separable in the green (around $550 \mathrm{~nm}$ ), NIR (941-1034 nm, 1064$1068 \mathrm{~nm}$ and 1140-1144 nm) and SWIR 1 (1532$1543 \mathrm{~nm}$ and $1677-1694 \mathrm{~nm}$ ) regions. When all the four forms were compared, lowest separability of species pairs could be noticed in SDS.

Factor analysis of statistically significant wavelengths under each of the four spectral forms helped in identifying the bands that were uncorrelated and contained maximum information for species discrimination. As per the outcomes of factor analysis of derivative spectra and CRRS, the red absorbance region (around $650 \mathrm{~nm}$ ), red edge region (680-720 nm), specific NIR bands (including 1152, 1156, 1159 and $1212 \mathrm{~nm}$ ) and SWIR 1 region played important role in species discrimination. The red edge region was found to be the most important wavelength region for discriminating vegetation canopies in some previous studies (Schmidt and Skidmore, 2003; Manevski et al., 2011; Prasad and Gnanappazham, 2016). The spectral variability in the red absorbance and red edge bands revealed that there was noteworthy difference in the amount of biochemical contents of leaves among the mangrove species, particularly the leaf pigments (Das et al., 2002; Zhang et al., 2014). The high separability in the NIR bands may be attributed to the difference in the amount of scattering due to multiple refractions and reflections at the boundary between cellular walls and air spaces in the mesophyll tissue. The spectral variability in the SWIR bands may be primarily accredited to absorption due to the achlorophyllous water storage tissue present in the leaves and different levels of leaf succulence (Wehe, 1964; Tomlinson, 1994; Panigrahy et al., 2012). However, thorough investigations are required for drawing firm conclusions regarding the wavelengths which could not be acquiescent with the spectral location(s) of mangrove leaf constituents.

As the J-M distance measure was a parametric method, it was necessary to decrease the number of spectral features (bands) prior to the calculations. More precisely, it was not feasible to calculate the $\mathrm{J}-\mathrm{M}$ distance using all the selected (after factor analysis) bands because of the singularity problem of matrix inversion. Hence, SDA of RS was performed to obtain a linear combination of few optimal bands which were then used for calculation of $\mathrm{J}-\mathrm{M}$ distance. The $\mathrm{J}-\mathrm{M}$ distances obtained for the species pairs showed that 91 pairs were discriminable in the spectral bands $(759,919$, 934, 940 and $948 \mathrm{~nm}$ ) considered in the present study. The square of the $\mathrm{J}-\mathrm{M}$ distance was found to 
be consistently above 1.9 (excluding one pair i.e. Bruguiera gymnorrhiza vs. C. decandra). Often the species under the same family visually exhibit similar phyllotaxy and branching pattern, thus, virtually the same canopy structure. This is supported by the fact that the spectral separabilities between the species of Rhizophoraceae, viz. B. gymnorrhiza, C. decandra and $R$. mucronata were found to be less and least in case of B. gymnorrhiza vs. C. decandra.

\section{Conclusions}

In the present work, it was studied that how non-parametric Mann-Whitney U test could be used for determining the significant (separable) bands for discriminating mangrove species. The derivative and continuum removed reflectance spectra together with reflectance spectra were used to identify the important spectral regions/ most consistent bands for discriminating the 14 species and 91 species pairs. The reflectance spectra alone were suitable for separability analysis in four pairs, but unsuitable for 15 pairs. In comparison to the reflectance spectra, the overall spectral separability increased in 24 pairs using continuum removed reflectance spectra. It was also noticed that in the first derivative spectra the separability had increased in the visible and SWIR 1 regions for four and three pairs, respectively. Besides, the first derivative spectra were unsuitable for discrimination of six pairs. In general, the red region, red edge region, specific NIR bands (including 759, 919, 934, 940, 948, 1152, 1156, 1159 and $1212 \mathrm{~nm}$ ) and shortwave infrared bands in the region 1503-1766 $\mathrm{nm}$ played important roles in mangrove species discrimination. The selection of crucial wavelengths for discrimination of mangrove species could be identified from different spectral forms using factor analysis. All the mangrove species dealt in the study were found to be spectrally distinguishable from each other in terms of the square of $\mathrm{J}-\mathrm{M}$ distance, excepting two species, namely, $B$. gymnorrhiza and $C$. decandra. The outcomes of the study also indicated the efficacy of the applied statistical tools for species discrimination.

\section{Acknowledgements}

The authors are obliged to the Director, NRSC for overall encouragement to take up this study. The authors express their sincere gratitude to the Principal Chief Conservator of Forests (PCCF), Director, Sundarban Biosphere Reserve) and other forest officials of West Bengal Forest Department for granting permission for field data collection in the forests. The authors are grateful to the field supporters for their help during in-situ data collection.

\section{References}

Ajai, Bahuguna, A., Chauhan, H.B., Sen Sarma, K., Bhattacharya, S., Ashutosh, S., Pandey, C.N., Thangaradjou, T., Gnanppazham, L., Selvam, V., Nayak, S.R., 2012. Mangrove inventory of India at community level. National Academy Science Letters 36, 67-77.

Apan, A. and Phinn, S., 2006. Special feature hyperspectral remote sensing. Journal of Spatial Science 52, 47-48.

Asner, G.P., 1998. Biophysical and biochemical sources of variability in canopy reflectance. Remote Sensing of Environment 64, 234-253.

Banerjee, L.K., Sastry, A.R.K., Nayar, M.P., 1989. Mangroves in India, identification manual. Botanical Survey of India, Kolkata, India.

Blasco, F., Aizpuru, M., 2002. Mangroves along the coastal stretch of the Bay of Bengal: present status. Indian Journal of Marine Sciences 31, 920.

Chakravortty, S., 2013. Application of hyperspectral data for development of mangrove species in the Sundarban delta. International Journal of Geomatics and Geosciences 4, 305-312.

Clark, R.N., King, T.V.V., Gorelick, N.S., 1987. Automatic continuum analysis of reflectance spectra. AIS Workshop. Pasadena.

Curran, P.J., 1989. Remote sensing of foliar chemistry. Remote Sensing of Environment 30, 271-278.

Das, A.B., Parida, A., Basak, U.C., Das, P., 2002. Studies on pigments, proteins and photosynthetic rates in some mangroves and mangrove associates from Bhitarkanika, Orissa. Marine Biology 141, 415-422.

Dawson, T.P., Curran, P.J., 1998. A new technique for interpolating the reflectance red edge position. International Journal of Remote Sensing 19, 2133-2139.

Demuro, M., Chisholm, L., 2003. Assessment of Hyperion for characterizing mangrove communities. International conference the AVIRIS. Pasadena.

Dineen, L.C., Blakesley, B.C., 1973. Algorithm AS 62: Generator for the sampling distribution of the Mann-Whitney U statistic. Applied Statistics 22, 269-273.

Giri, C., Ochieng, E., Tieszen, L.L., Zhu, Z., Singh, A., Loveland, T., Masek, J., Duke, N., 2011. Status and distribution of mangrove forests of the world using earth observation satellite data. Global Ecology and Biogeography 20, 154-159.

Giri, S., Mukhopadhyay, A., Hazra, S., Mukherjee, S., Roy, D., Ghosh, S., Ghosh, T., Mitra, D., 2014. A study on abundance and distribution of mangrove species in Indian Sundarban using 
remote sensing technique. Journal of Coastal Conservation 18, 359-367.

Green, P.E., Caroll, J.D., 1978. Mathematical tools for applied multivariate analysis. Academic Press, New York.

Held, A., Ticehurst, C., Lymburner, L., Williams, N., 2003. High resolution mapping of tropical mangrove ecosystems using hyperspectral and radar remote sensing. International Journal of Remote Sensing 24, 2739-2759.

Hirano, A., Madden, M, Welch, R., 2003. Hyperspectral image data for mapping wetland vegetation. Wetlands 23, 436-448.

Holmgren, P.K., Thuresson, T.T., 1998. Satellite remote sensing for forestry planning. Journal of Forest Research 13, 90-110.

Huitric, M., Folke, C., Kautsky, N., 2002. Development and government policies of the shrimp farming industry in Thailand in relation to mangrove ecosystems. Ecological Economics 40, 441-455.

Jensen, J.R., 2005. Introductory digital image processing: a remote sensing perspective, 3rd ed. Pearson Prentice Hall, Sydney, NSW, Australia.

Jusoff, K., 2006. Individual mangrove species identification and mapping in Port Klang using airborne hyperspectral imaging. Journal of Sustainability Science and Management 1, 27-36.

Kamal, M., Phinn, S., 2011. Hyperspectral data for mangrove species mapping: a comparison of pixel-based and object-based approach. Remote Sensing 3, 2222-2242.

Kamaruzaman, J., Kasawani, I., 2007. Imaging spectroscopy on mangrove species identification and mapping in Malaysia. WSEAS Transactions on Biology and Biomedicine 4, 118-126.

Knapp, A.K., Carter, G.A., 1998. Variability in leaf optical properties among 26 species from a broad range of habitats. American Journal of Botany 85, 940-946.

Koedsin, W., Vaiphasa, C., 2013. Discrimination of tropical mangroves at the species level with EO-1 Hyperion data. Remote Sensing 3, 878-928.

Kuenzer, C., Bluemel, A., Gebhardt, S., Quoc, T.V., , Dech, S., 2011. Remote sensing of mangrove ecosystems: a review. Remote Sensing 3, 878928.

Kumar, L., Schmidt, K., Durry, S., Skidmore, A.K., 2001. Imaging spectrometry and vegetation science, in: Van de Meer F., De Jong S.M. (Eds), Imaging Spectrometry. Kluwer Academic Press, Dordrecht, pp 111-155.

Kumar, T., Panigrahy, S., Kumar, P., Parihar, J.S., 2013. Classification of floristic composition of mangrove forests using hyperspectral data: case study of Bhitarkanika National Park. Journal of Coastal Conservation 17, 121-132.
Manevski, K., Manakos, I., Petropoulos, G.P., Kalaitzidis, C., 2011. Discrimination of common Mediterranean plant species using field spectroradiometry. International Journal of Applied Earth Observation and Geoinformation 13, 922-933.

Manjunath, K.R., Kumar, T., Kundu, N., Panigrahy, S., 2013. Discrimination of mangrove species and mudflat classes using in situ hyperspectral data: a case study of Indian Sundarbans. GIScience and Remote Sensing 50, 400-417.

Martin, M.E., Newman, S.D., Aber, J.D., Congalton, R.G., 1998. Determining forest species composition using high spectral resolution remote sensing data. Remote Sensing of Environment 65, 249-254.

Mutanga, O., Skidmore, A., 2007. Red edge shift and biochemical content in grass canopies. ISPRS Journal of Photogrammetry and Remote Sensing $62,34-42$.

Norusis, M., 2004. SPSS 13.0 Statistical procedures companion. Prentice Hall, Upper Saddle River, NJ.

Panigrahy, S., Kumar, T., Manjunath, K.R., 2012. Hyperspectral leaf signature as an added dimension for species discrimination: case study of four tropical mangroves. Wetlands Ecology and Management 20, 101-110.

Prasad, K.A., Ganappazham, L., 2014. Species discrimination of mangrove using derivative spectral analysis. ISPRS Annals of the Photogrammetry, Remote Sensing and Spatial Information Sciences 8, 45-52.

Prasad, K.A., Gnanappazham, L., 2016. Multiple statistical approaches for the discrimination of mangrove species of Rhizophoraceae using transformed field and laboratory hyperspectral data. Geocarto International 31, 891-912.

Prasad, K.A., Gnanappazham, L., Selvam, V., Ramasubramanian, R., Kar, C.S., 2015. Developing a spectral library of mangrove species of Indian east coast using field spectroscopy. Geocarto International 30, 580-599.

Reddy, C.S., Pattanaik, C., Murthy, M.S.R., 2007. Assessment and monitoring of mangroves of Bhitarkanika Wildlife Sanctuary, Orissa, India using remote sensing and GIS. Current Science 92, 1409-1415.

Richards, J.A., Jia, X., 2006. Remote sensing digital image analysis: an introduction, 4th ed. Springer, Germany.

Schmidt, K.S., Skidmore, A.K., 2003. Spectral discrimination of vegetation types in a coastal wetland. Remote Sensing of Environment 85, 92 108.

Snedaker, S.C., 1982. Mangrove species zonation: why? in: Sen D.N., Rajpurohit, K.S. (Eds.), Tasks 
for Vegetation Science, 2. Dr W. Junk Publishers, The Hague, pp 111-125.

State of Forest Report. 2017. Forest Survey of India. Ministry of Environment and Forests, Dehradun, India. http://fsi.nic.in/forest-report-2017.

Sun, Y., Liu, X., Wu, Y., Liao, C., 2008. Identifying hyperspectral characters of wetland species using in situ data. The International Archives of the Photogrammetry, Remote Sensing and Spatial Information Sciences 37, 459-466.

Thomas, V., Treitz, P., Jelinski, D., Miller, J., Lafleur, P., Mccaughey, J.H., 2002. Image classificaiton of a northern peatland complex using spectral and plant community data. Remote Sensing of Environment 84, 83-99.

Tomlinson, P.B., 1994. The botany of mangroves. Cambridge University Press, Cambridge.

Tsai, F., Philpot, W., 1998. Derivative analysis of hyperspectral data. Remote Sensing of Environment 66, 41-51.

Vaiphasa, C., Ongsomwang, S., Vaiphasa, T., Skidmore, A.K., 2005. Tropical mangrove species discrimination using hyper spectral data: a laboratory study. Estuarine Coastal Shelf Science 65, 371-379.

Van Der Meer, F., De Jong, S., Bakker, W., 2001. Imaging spectrometry: basic analytical techniques, in: Van Der Meer, F., De Jong S (Eds.), Imaging Spectrometry: basic principles and prospective applications. Kluwer Academic Publishers, Dordrecht, pp. 17-61.

Wang, L.E., Sousa, W.P., 2009. Distinguishing mangrove species with laboratory measurements of hyperspectral leaf reflectance. International Journal of Remote Sensing 30, 1267-1281.

Wehe, V., 1964. Beitrage zur Okologie der mittle und westeuropaischen Salzgetation (Gezeitenkusten). Biet Biol Pflanzen 39, 189-237.

Zhang, C., Kovacs, J.M., Liu, Y., Flores-Verdugo, F., Flores-De-Santiago, F., 2014. Separating mangrove species and conditions using laboratory hyperspectral data: a case study of a degraded mangrove forest of the Mexican Pacific. Remote Sensing 6, 11673-11688. 